\title{
Separability of Stokes Equations in Axisymmetric Geometries
}

\author{
Maria Hadjinicolaou1, Eleftherios Protopapas² \\ ${ }^{1}$ Applied Mathematics Laboratory, School of Science and Technology, Hellenic Open University, 167 R. Feraiou St., \\ GR-26 222 Patras, Greece \\ ${ }^{2}$ School of Applied Mathematical and Physical Sciences, National Technical University of Athens, 9 Heroon Polytechneiou St., \\ GR-15 780 Athens, Greece \\ Email: hadjinicolaou@eap.gr, lprotopapas@math.ntua.gr
}

How to cite this paper: Hadjinicolaou, $M$. and Protopapas, E. (2020) Separability of Stokes Equations in Axisymmetric Geometries. Journal of Applied Mathematics and Physics, 8, 315-348.

https://doi.org/10.4236/jamp.2020.82026

Received: January 2, 2020

Accepted: February 23, 2020

Published: February 26, 2020

Copyright (C) 2020 by author(s) and Scientific Research Publishing Inc. This work is licensed under the Creative Commons Attribution International License (CC BY 4.0).

http://creativecommons.org/licenses/by/4.0/

\begin{abstract}
For Stokes flow in non spherical geometries, when separation of variables fails to derive closed form solutions in a simple product form, analytical solutions can still be obtained in an almost separable form, namely in semiseparable form, R-separable form or R-semiseparable form. Assuming a stream function $\psi$, the axisymmetric viscous Stokes flow is governed by the fourth order elliptic partial differential equation $E^{4} \psi=0$ where $E^{4}=E^{2} O E^{2}$ and $E^{2}$ is the irrotational Stokes operator. Depending on the geometry of the problem, the general solution is given in one of the above separable forms, as series expansions of particular combinations of eigenfunctions that belong to the kernel of the operator $E^{2}$. In the present manuscript, we provide a review of the methodology and the general solutions of the Stokes equations, for almost any axisymmetric system of coordinates, which are given in a ready to use form. Furthermore, we present necessary and sufficient conditions that are serving as criterion for identifying the kind of the separation the Stokes equation admits, in each axisymmetric coordinate system. Additionally, as an illustration of the usefulness of the obtained analytical solutions, we demonstrate indicatively their application to particular Boundary Value Problems that model medical problems.
\end{abstract}

\section{Keywords}

Axisymmetric Creeping Flow, R-Separation, Semiseparation, R-Semiseparation, Irrotational Flow, Analytical Solution

\section{Introduction}

Separation of variables is undoubtedly among the most powerful methods for 
solving analytically partial differential equations (PDEs). It can be applied to problems regardless of the number of dimensions and provides both, qualitative and quantitative information for the behavior of the solution in the whole domain. This is extremely useful when studying physical, biological, medical and engineering problems or problems where the asymptotic behavior or limiting cases can be reached in a straight forward manner through analytical methods, eliminating the need of imposing further assumptions, as a numerical treatment of the problem should require. A key aspect for obtaining separable solutions of Boundary Value Problems (BVPs) in 3-D, concerns the identification and the reflection of the geometrical characteristics of the problem to the choice of the suitable orthogonal curvilinear system of coordinates, i.e. the one for which the physical boundary of the problem coincides to one of the coordinate surfaces. Expressing the partial differential operator in the particular system, the derivation of separable solutions depends then on the analytical solvability of the associate ordinary differential equations (ODEs), in which the partial differential equation decomposes. The orthogonality property of the eigenfunctions that belong to each of the solution subspaces, quantifies their "contribution" to the exact solution of the BVP at hand.

Moon and Spencer in [1] and Morse and Feshbach in [2] have investigated extensively, the conditions under which separable or R-separable solution of the Laplace and the Helmholtz equations can be obtained, in various orthogonal curvilinear systems of coordinates. These equations are used to model potential and wave problems and their separability is very well studied. On the contrary, the separability of Stokes equations, only recently has been studied exhaustively by the authors, in any axisymmetric curvilinear system [3].

Stokes equations describe the viscous axisymmetric flow of a Newtonian fluid [4] and may describe the flow through porous media [5] [6] [7] or the flow of biological fluids, e.g. blood plasma [8] and many more. Stokes flow is described mathematically through a system of two differential equations employing the velocity and the pressure field. Specifically, in axisymmetric cases, these quantities can be calculated through a scalar function $\psi$, namely the stream function, which satisfies the fourth order elliptic partial differential Equation (PDE) $E^{4} \psi=0$ where $E^{4}=E^{2} o E^{2}$ and $E^{2}$ is the irrotational Stokes operator.

The fact that the equation $E^{2} \psi=0$ separates variables in spherical coordinates is known almost 170 years ago [5]. The general solution of Stokes flow in the spherical coordinate system has been used for solving several problems. Sampson [9] moving forward his own work used the general solution to provide the solution of the flow past an approximate sphere, while Kim [10] provided the analytical solution for the flow past three spheres. Sankar [11] derived solutions for flows in and around a sphere or between concentric spheres.

Although many attempts had been made for deriving solutions in other than the spherical coordinate system, such as the prolate and the oblate spheroidal ones, closed form solutions of Stokes equations were obtained, only 150 years later, and recently, a solution method and complete solutions expansions were 
obtained in the inverted spheroidal systems. Lastly, the authors identified and proved the necessary and sufficient conditions for the separation and R-separation of the Stokes operator in any axisymmetric system of coordinates, augmented this way the theoretical knowledge on the field and providing ready to use expansions for solving analytically boundary value problems [3].

More precisely, Oberbeck [12] in 1876, using Cartesian coordinates, derived a solution for the Stokes flow in an unbounded fluid due to the steady translation of an ellipsoid. Sampson in 1891 [9] obtained a partial solution of the Stokes flow along the main axis of a translating spheroid in an unbounded fluid, using spheroidal coordinates. Payne and Pell in 1960 [13] derived a solution for Stokes flow around a spheroid. Happel and Brenner [5], provided a solution for the axisymmetric viscous flow around a single spheroid with different boundary conditions using an ad-hoc technique, which seemed to be adequate for solving approximately engineering problems, although a general solution of the governing fourth order partial differential equation was not known. Coutelieris et al. [14] [15] used spheroid-in-cell models to study the mass transfer of a swarm of spheroidal (prolate or oblate) adsorbers in Stokes flow. Ken and Chang [16] studied the motion of a spheroidal particle freely suspended in a gaseous medium with a uniform temperature with small Peclet and Reynolds numbers and in [17] studied the Stokes flow caused by a rigid spheroidal particle in a viscous fluid with slip boundary condition. Zlatanovksi [18] used the Brinkman model to solve Stokes flow past a porous prolate spheroidal particle while Deo and Datta [19] solved Stokes flow past a fluid prolate spheroid. Moreover, Deo and Gupta [20] derived the solution of Stokes flow of an incompressible viscous fluid past a swarm of porous approximately spheroidal particles with Kuwabara boundary condition.

Dassios et al. [6] using linear algebra theory, derived the complete solution of Stokes equation in spheroidal cell, by introducing the concept of semiseparation of variables. Particularly, they derived the 0 -eigenspace and the generalized 0 -eigenspace of the operator $E^{2}$ in the spheroidal coordinates which is consisted of eigenfunctions in separable form, given in terms of products of Gegenbauer functions of the first and the second kind. The complete representation of the solution space of $E^{4} \psi=0$ is obtained as a sum of series expansion of these separable eigenfunctions and the series expansions of the generalized eigenfunctions which are given in terms of mixed order Gegenbauer functions. An extensive review of the relative literature can be found in [6]. Dassios and Vafeas in [21] rearranged these expansions in a different way aiming to provide a more convenient expansion. Deo and Tiwari in 2008 [22] derived the complete solution of the equation $E^{2} \psi=0$, in bispherical and toroidal coordinate systems, while Hadjinicolaou and Protopapas proved the R-separation of Stokes equation in the inverted prolate and oblate spheroidal coordinate systems [23] [24] [25], which further expands our tools to treat Stokes flow problems around non convex bodies.

Specifically, the authors proved that Stokes equation $E^{2} \psi=0$, in the inverted 
prolate coordinate system [23] [24] and in the inverted oblate coordinate system [25], R-separates variables and they derived the corresponding eigensolutions. Aiming to obtain the solution of the $E^{4} \psi=0$, they used the concept of the semiseparation of variables and developed an algorithm through which the generalized eigenfunctions of the kernel of $E^{2}$, are given through recurrence relations, since the generalized eigenfunctions could not be expressed in a closed form. The eigenfunctions of the 0-eigenspace are expressed as products of $\mathrm{Ge}$ genbauer functions divided by the Euclidean distance $r$, while the generalized 0 -eigenspace is consisted of combinations of products of Gegenbauer functions, in semiseparable form, divided by the third power of the Euclidean distance, $r^{3}$.

This solution expansion was utilized by Dassios et al. [8] to study the flow past a red blood cell, modeled as an inverted prolate spheroid, while Hadjinicolaou et al. expanded this model to treat the sedimentation of a red blood cell [26] and also the blood plasma flow around two aggregated low density lipoproteins [27] and the translation of two aggregated low density lipoproteins within blood plasma [28]. These results are demonstrated in Section 6.

To this end, departing from the spheroidal geometries, in [3] the authors investigated, formulated and proved the necessary and sufficient conditions for the separation or the R-separation of $E^{2} \psi=0$ in any axisymmetric system of coordinates, and provided a road map for deriving the relative eigenfunctions. In the case of R-separability the exact form of the function $R$ was identified as well. They also proved the general statement that if Stokes equation separates variables in a system then it R-separates variables in the inverted one, while if it $\mathrm{R}$-separates variables, it can also R-separates variables in the corresponding inverted system of coordinates, if an extra condition is satisfied.

The structure of the manuscript is as follows. In Section 2, the physical and mathematical background is given, while in Section 3, we present the necessary and sufficient conditions for the separability of Stokes equation $E^{2} \psi=0$. In Section 4, we review the different kinds of separation that the equations $E^{2} \psi=0, E^{4} \psi=0$ admit in spherical and spheroidal geometries and in Section 5, we show results, regarding the irrotational flow in other axisymmetric systems of coordinates. In Section 6, we display applications in Biology, while in Section 7 we discuss some key points of the obtained results.

\section{Rotational and Irrotational Flow}

The steady flow of an incompressible fluid around particles where the viscous forces dominate over the inertial ones is called Stokes flow since it was first studied by sir George Stokes [4]. When particles are embedded in a fluid domain $\Omega \subseteq \mathbb{R}^{3}$, Stokes flow is described [5] as

$$
\begin{gathered}
\mu \Delta \boldsymbol{v}(\boldsymbol{r})=\nabla P(\boldsymbol{r}), \boldsymbol{r} \in \Omega, \\
\nabla \cdot \boldsymbol{v}(\boldsymbol{r})=0, \boldsymbol{r} \in \Omega,
\end{gathered}
$$

where $\boldsymbol{r}$ is the position vector, $\boldsymbol{v}(\boldsymbol{r})$ is the velocity field, $P(\boldsymbol{r})$ is the pres- 
sure field and $\mu$ is the shear viscosity.

In the axisymmetrical case the flow is described by a function $\psi$, namely stream function, which satisfies the fourth order partial elliptic equation [5]

$$
E^{4} \psi=0,
$$

where $E^{4}=E^{2} O E^{2}$ is the Stokes bi-stream operator and $E^{2}$ is the Stokes operator, which describes the irrotational flow

$$
E^{2} \psi=0 .
$$

Since the flow is assumed as an axisymmetric one, Stokes operator has to be expressed in an axisymmetric coordinate system.

Any axisymmetric system of coordinates $\left(q_{1}, q_{2}, \varphi\right), \varphi \in[0,2 \pi)$ is defined via the relations

$$
\left\{\begin{array}{l}
x_{1}=\rho\left(q_{1}, q_{2}\right) \cos \varphi \\
x_{2}=\rho\left(q_{1}, q_{2}\right) \sin \varphi \\
x_{3}=z\left(q_{1}, q_{2}\right)
\end{array}\right.
$$

where $\left(x_{1}, x_{2}, x_{2}\right)$ denote a point in the Cartesian coordinates. The metric coefficients $h_{1}, h_{2}$ and the radial cylindrical coordinate $\varpi$ [5] are

$$
h_{1}=\frac{1}{\sqrt{\left(\frac{\partial \rho}{\partial q_{1}}\right)^{2}+\left(\frac{\partial z}{\partial q_{1}}\right)^{2}}}, h_{2}=\frac{1}{\sqrt{\left(\frac{\partial \rho}{\partial q_{2}}\right)^{2}+\left(\frac{\partial z}{\partial q_{2}}\right)^{2}}}, \varpi=\left|\rho\left(q_{1}, q_{2}\right)\right| \text {, }
$$

while Stokes operator, $E^{2}$, assumes the form

$$
E^{2}=h_{1} h_{2} \varpi\left[\frac{\partial}{\partial q_{1}}\left(\frac{h_{1}}{h_{2} \varpi} \frac{\partial}{\partial q_{1}}\right)+\frac{\partial}{\partial q_{2}}\left(\frac{h_{2}}{h_{1} \varpi} \frac{\partial}{\partial q_{2}}\right)\right] .
$$

The knowledge of the stream function $\psi$ enables us to derive significant hydrodynamic quantities, such as the velocity components

$$
\left\{\begin{array}{l}
u_{q_{1}}=-\frac{h_{2}}{\varpi} \frac{\partial \psi}{\partial q_{2}} \\
u_{q_{2}}=\frac{h_{1}}{\varpi} \frac{\partial \psi}{\partial q_{1}}
\end{array}\right.
$$

the pressure field $P$

$$
\left\{\begin{array}{l}
\frac{\partial P}{\partial q_{1}}=-\frac{h_{2}}{h_{1}} \frac{\mu}{\varpi} \frac{\partial\left(E^{2} \psi\right)}{\partial q_{2}} \\
\frac{\partial P}{\partial q_{2}}=-\frac{h_{1}}{h_{2}} \frac{\mu}{\varpi} \frac{\partial\left(E^{2} \psi\right)}{\partial q_{1}}
\end{array}\right.
$$

the drag force

$$
F_{z}=\pi \mu \int \varpi^{3} \frac{\partial}{\partial n}\left(\frac{E^{2} \psi}{\varpi^{2}}\right) \delta s
$$

and the drag coefficient 


$$
C_{d}=\frac{2 F_{z}}{\rho U^{2} A},
$$

where $U$ is the particle speed, $A$ is the cross sectional area, $\rho$ is the fluid density. Moreover, we can derive the settling terminal velocity $U_{\infty}$. This is the velocity of a particle when the gravitational force acting on it and the drag force become equal, and given via the equation

$$
F_{z}=\left(\rho^{\prime}-\rho\right) g V,
$$

where $\rho^{\prime}$ is the mean particle density, $g$ is the local acceleration of gravity vector and $V$ is the particle's volume.

\section{Necessary and Sufficient Conditions for the Separation and the R-Separation of Stokes Equation $E^{2} \psi=0$}

Among the most useful methods on solving a PDE is the separation (and $\mathrm{R}$-separation of variables). In both cases, the unknown function decomposes the PDE in ODEs. In the simple separation of variables we assume that the unknown function can be written as a product of functions of one variable, while in the case of the R-separation the product is assumed to be multiplied by a function $R$ of at least two variables (not in a product form). In what follows we present the necessary theory: two theorems and a lemma, that we need in order to examine whether the Stokes operator $E^{2}$ separates or R-separates variables in axisymmetric system of coordinates [3].

Theorem 1. If $\left(q_{1}, q_{2}, \varphi\right)$ is an axisymmetric system of coordinates with metric coefficients $h_{1}, h_{2}$ and radial cylindrical coordinate $\varpi$, the Stokes equation separates variables if and only if there exist functions

$$
\begin{aligned}
f_{1}\left(q_{1}\right), f_{2}\left(q_{2}\right), F_{1}\left(q_{1}\right), F_{2}\left(q_{2}\right) \text { such as } & \\
\frac{h_{1}}{h_{2} \varpi} & =f_{1} f_{2}
\end{aligned}
$$

and

$$
\frac{h_{2}}{h_{1} \varpi}=F_{1} F_{2} .
$$

Theorem 2. If $\left(q_{1}, q_{2}, \varphi\right)$ is an axisymmetric system of coordinates with metric coefficients $h_{1}, h_{2}$ and radial cylindrical coordinate $\varpi$, the Stokes equation $R$-separates variables if and only if there exist functions

$$
\begin{gathered}
f_{1}\left(q_{1}\right), f_{2}\left(q_{2}\right), F_{1}\left(q_{1}\right), F_{2}\left(q_{2}\right), R\left(q_{1}, q_{2}\right), R_{1}\left(q_{1}\right), R_{2}\left(q_{2}\right) \text { such that } \\
\frac{h_{1}}{h_{2} \varpi}=R^{2} f_{1} f_{2}, \\
\frac{h_{2}}{h_{1} \varpi}=R^{2} F_{1} F_{2}, \\
\frac{1}{F_{1}} \frac{\partial}{\partial q_{1}}\left(f_{1} \frac{\partial R}{\partial q_{1}}\right)+\frac{1}{f_{2}} \frac{\partial}{\partial q_{2}}\left(F_{2} \frac{\partial R}{\partial q_{2}}\right)=R\left(R_{1}+R_{2}\right),
\end{gathered}
$$


where $R\left(q_{1}, q_{2}\right) \neq g_{1}\left(q_{1}\right) g_{2}\left(q_{2}\right)$.

Lemma 1. Let an axisymmetric system of coordinates $\left(q_{1}, q_{2}, \varphi\right)$ with metric coefficients $h_{1}, h_{2}$, radial cylindrical coordinate $\varpi$ and the corresponding system of coordinates under the inversion with respect to a sphere of radius $b>0$ having metric coefficients $h_{1}^{\prime}, h_{2}^{\prime}$ and radial cylindrical coordinate $\varpi^{\prime}$, then the following relations, interconnecting the metric coefficients hold true.

$$
\begin{aligned}
h_{1}^{\prime} & =\frac{r^{2}}{b^{2}} h_{1}, \\
h_{2}^{\prime} & =\frac{r^{2}}{b^{2}} h_{2}, \\
\varpi^{\prime} & =\frac{b^{2}}{r^{2}} \varpi .
\end{aligned}
$$

These two theorems formulate separability conditions of Stokes operator in any axisymmetric systems of coordinates. The results use geometrical characteristics of the system, which are the metric coefficients $h_{1}, h_{2}$ and the radial cylindrical coordinate $\varpi$. Then the following steps have to be applied. Calculating the quantities $\frac{h_{1}}{h_{2} \varpi}, \frac{h_{2}}{h_{1} \varpi}$ we first examine whether conditions (13), (14) hold. If these are true we assume simple separation of variables. Else we investigate whether (15), (16) hold. These conditions allow us to identify a function $R$, such that Equation (17) is also satisfied and thus R-separability is attained. Moreover, by employing the lemma, we interrelate the conditions needed for separation in an axisymmetric system with those needed for the separation in the corresponding inverted one. Specifically:

- if Stokes equation separates variables in an axisymmetric system of coordinates, then Stokes equation $\mathrm{R}$-separates variables in the corresponding inverted system of coordinates with $R\left(q_{1}, q_{2}\right)=r$, where $r$ is the Euclidean distance, expressed in the parameters of the particular coordinate system.

- if Stokes equation R-separates variables in an axisymmetric system of coordinates, then Stokes equation also R-separates variables in the corresponding inverted system of coordinates if (17) is also true.

These results are of a great importance since any solution of the equation $E^{4} \psi=0$, describing Stokes flow in any axisymmetric system of coordinates, belongs either to the kernel space of $E^{2}$, or to the corresponding generalized eigenspace of $E^{2}$. The conditions under which $E^{2} \psi=0$ separates or R-separates variables are fully investigated in [3].

\section{Separability of $E^{2} \psi=0$ and $E^{4} \psi=0$ in Spherical and Spheroid Geometries}

In this section, we present results regarding the solutions of the equations 
equations $E^{2} \psi=0, E^{4} \psi=0$ obtained through separation and the R-separation of variables and also through the so-called semiseparation and the R-semiseparation of variables, in the spheroidal coordinate systems.

\subsection{Separation in Spherical Geometry}

The most common geometry employed when studying flow around particles is the spherical one. Stokes operator [5] in spherical coordinates system $(r, \zeta, \phi), r>0, \zeta \in[-1,1], \phi \in[0,2 \pi)$ is

$$
E^{2}=\frac{\partial^{2}}{\partial r^{2}}+\frac{1-\zeta^{2}}{r^{2}} \frac{\partial^{2}}{\partial \zeta^{2}}
$$

where every point $\left(x_{1}, x_{2}, x_{3}\right)$ in the Cartesian coordinates [1] is expressed as

$$
\left(x_{1}, x_{2}, x_{3}\right)=\left(r \sqrt{1-\zeta^{2}} \cos (\varphi), r \sqrt{1-\zeta^{2}} \sin (\varphi), r \zeta\right) .
$$

Equation $E^{2} \psi=0$ separates variables in spherical geometry [5] and the solution space consists of products of functions of each one of the independent variables the radial and the angular ones, which are

$$
r^{n} G_{n}(\zeta), r^{n} H_{n}(\zeta), r^{-n+1} G_{n}(\zeta), r^{-n+1} H_{n}(\zeta),
$$

where $G_{n}, H_{n}$ are the Gegenbauer functions of the first and the second kind, respectively [29]. The Gegenbauer functions $G_{n}(\zeta), H_{n}(\tau)$ are related to Legendre polynomial and functions [5] [29].

In Figure 1 and Figure 2, we depict streamlines in spherical geometry for the eigenfunctions $r^{-1} H_{2}(\zeta), r^{-2} H_{3}(\zeta)$ that satisfy $E^{2} \psi=0$.

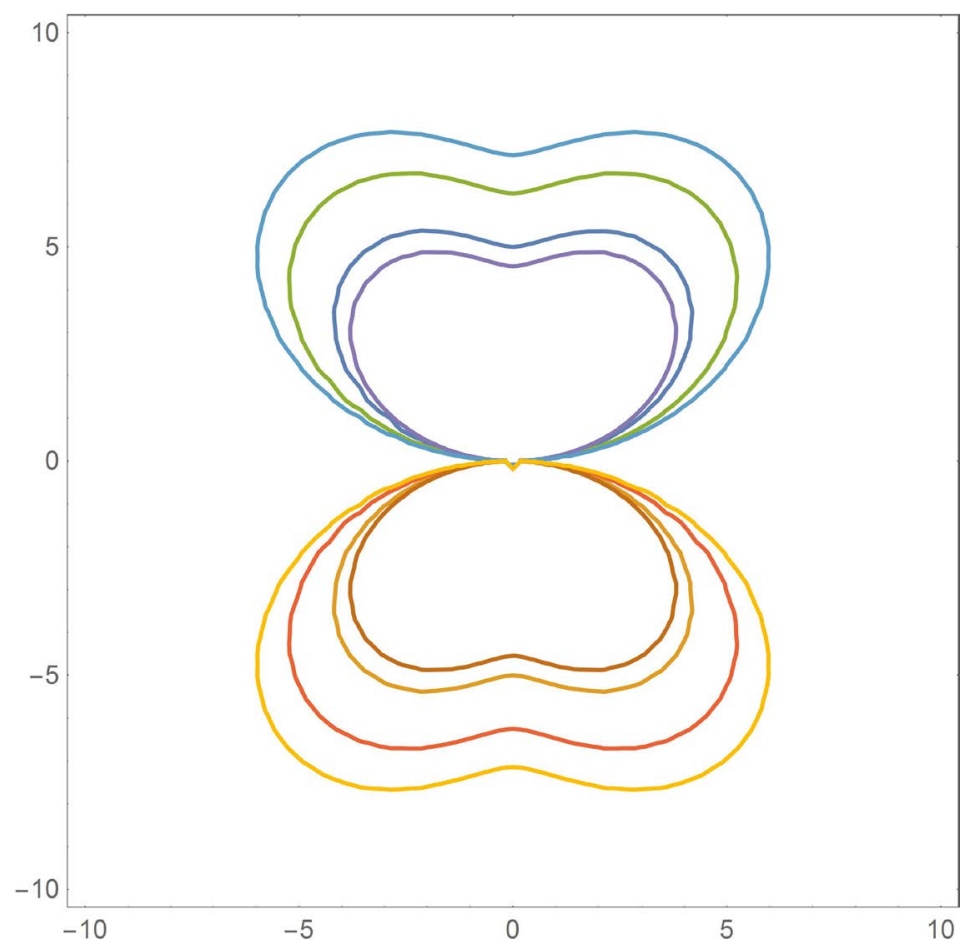

Figure 1. Streamlines for $r^{-1} H_{2}(\zeta)$ in spherical geometry. 


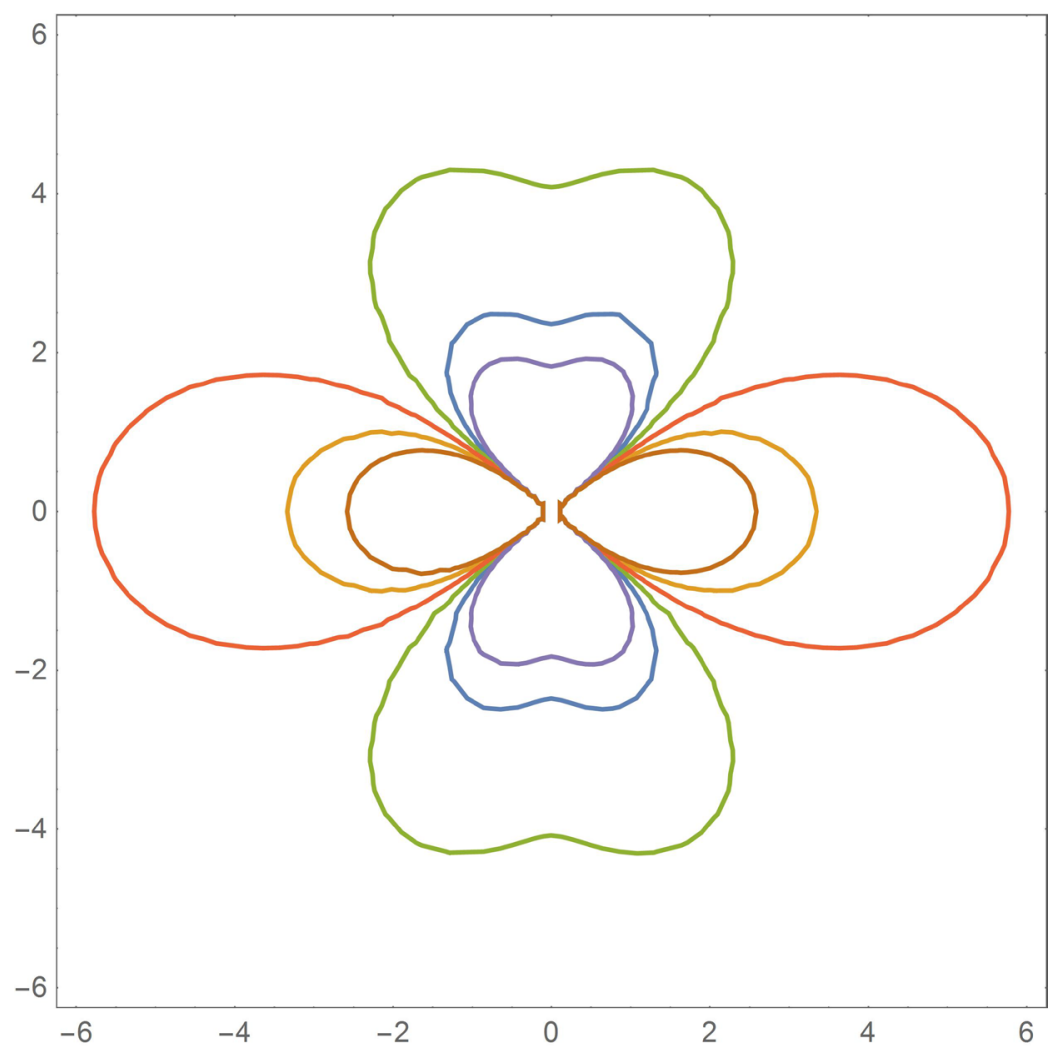

Figure 2. Streamlines for $r^{-2} H_{3}(\zeta)$ in spherical geometry.

In order to calculate the solution of $E^{4} \psi=0$ the concept of the generalized eigenfunctions is used and the relative methodology is processed. Accordingly, one needs to derive the functions $\psi_{g}$ that satisfy $E^{2} \psi_{g}=W$, where $W$ is a solution of $E^{2} \psi=0$. The functions $\psi_{g}$ are the generalized eigenfunctions of $E^{2}$ which are of the form

$$
r^{n+2} G_{n}(\tau), r^{n+2} H_{n}(\tau), r^{-n+3} G_{n}(\tau), r^{-n+3} H_{n}(\tau) .
$$

In Figure 3 and Figure 4, we present streamlines in spherical geometry for the generalized eigenfunctions $r^{4} H_{2}(\zeta), r^{5} G_{3}(\zeta)$ of $E^{2}$.

Taking into account (23), (24) we conclude that $E^{4} \psi=0$ also separates variables.

\subsection{Separation and Semiseparation in Prolate Spheroidal Geometry}

In the prolate system of coordinates $(\tau, \zeta, \phi), \tau \geq 1, \zeta \in[-1,1], \phi \in[0,2 \pi)$ any point $\left(x_{1}, x_{2}, x_{3}\right)$ is defined as [1]

$$
\left(x_{1}, x_{2}, x_{3}\right)=\left(c \sqrt{\tau^{2}-1} \sqrt{1-\zeta^{2}} \cos (\varphi), c \sqrt{\tau^{2}-1} \sqrt{1-\zeta^{2}} \sin (\varphi), c \tau \zeta\right),
$$

where $c>0$ is the semifocal distance and Stokes operator [6] is

$$
E^{2}=\frac{1}{c^{2}\left(\tau^{2}-\zeta^{2}\right)}\left[\left(\tau^{2}-1\right) \frac{\partial^{2}}{\partial \tau^{2}}+\left(1-\zeta^{2}\right) \frac{\partial^{2}}{\partial \zeta^{2}}\right] \text {. }
$$




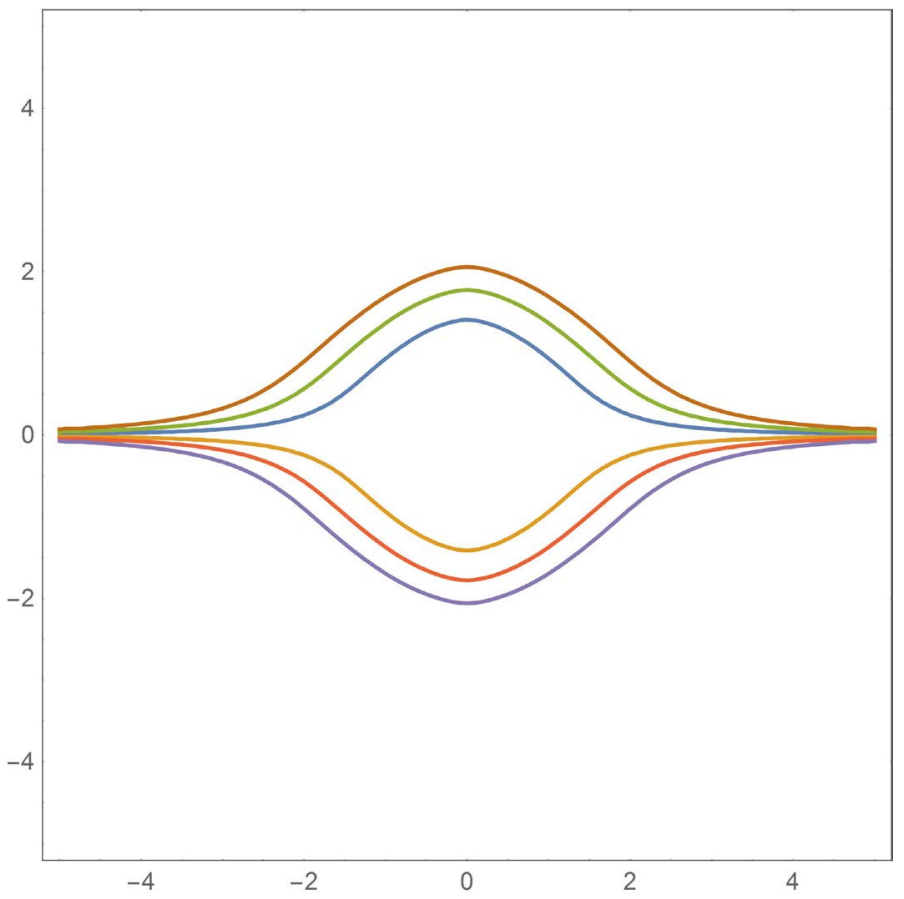

Figure 3. Streamlines for $r^{4} H_{2}(\zeta)$ in spherical geometry.

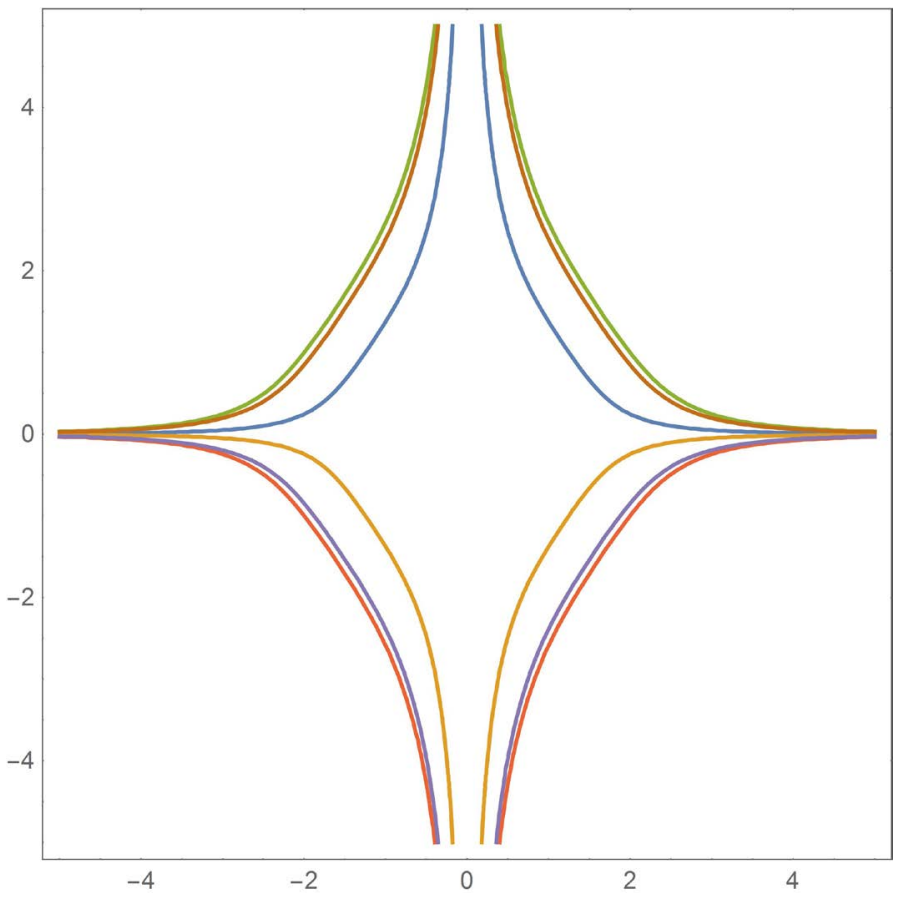

Figure 4. Streamlines for $r^{5} G_{3}(\zeta)$ in spherical geometry.

Equation $E^{2} \psi=0$ separates variables and the obtained eigenfunctions are

$$
G_{n}(\tau) G_{n}(\zeta), G_{n}(\tau) H_{n}(\zeta), H_{n}(\tau) G_{n}(\zeta), H_{n}(\tau) H_{n}(\zeta) .
$$

In Figure 5 and Figure 6, we depict sample streamlines in prolate geometry for the eigenfunctions $H_{2}(\tau) G_{2}(\zeta), H_{3}(\tau) G_{3}(\zeta)$ that satisfy $E^{2} \psi=0$. Using 


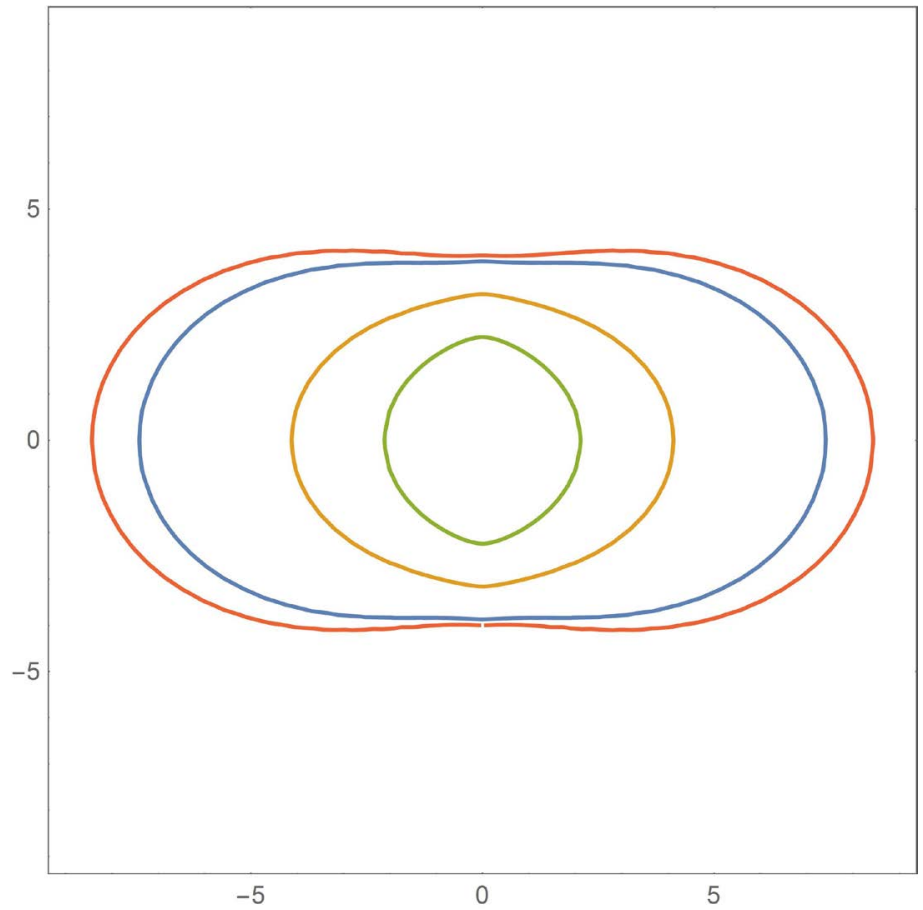

Figure 5. Streamlines for $H_{2}(\tau) G_{2}(\zeta)$ in prolate spheroid.

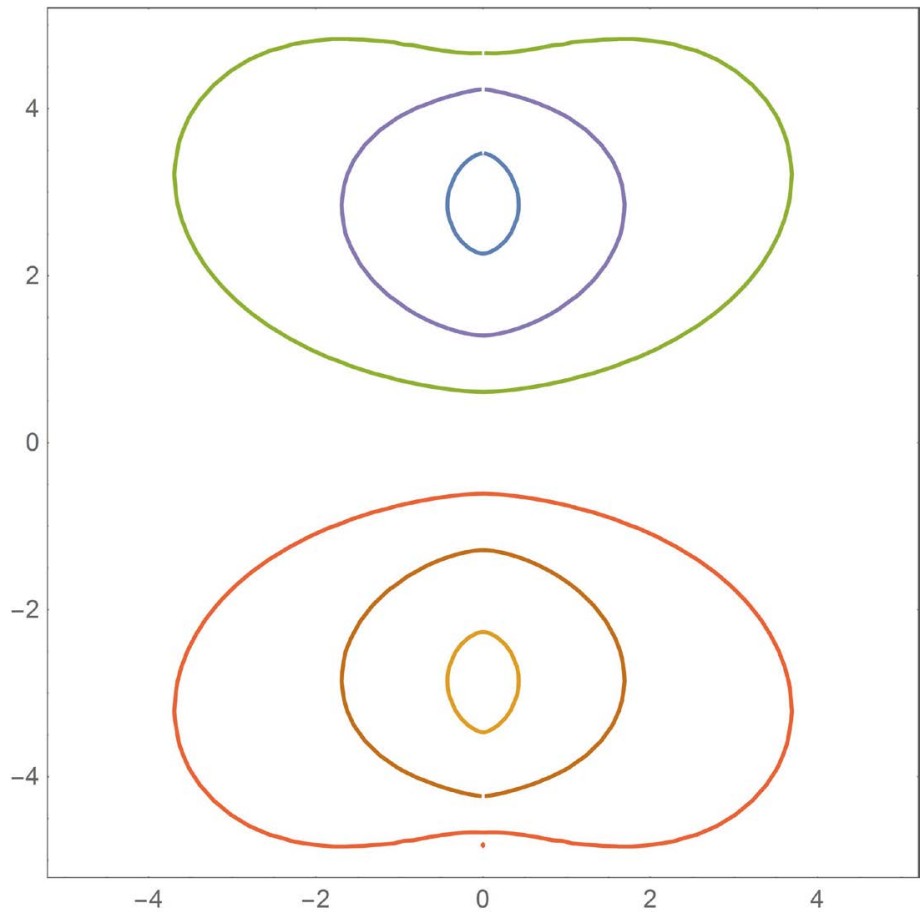

Figure 6. Streamlines for $H_{3}(\tau) G_{3}(\zeta)$ in prolate spheroid.

the methodology that we followed in the spherical case and taking into account that the prolate spheroid degenerates to a sphere when the semifocal distance tends to zero, we obtain the generalized eigenfunctions of Stokes operator as products of Gegenbauer functions of mixed order, such as: 


$$
\begin{gathered}
\Omega_{1}(\tau, \zeta)=-\frac{1}{3} G_{0}(\tau) G_{3}(\zeta)+G_{2}(\tau) G_{1}(\zeta), \\
\Omega_{2}(\tau, \zeta)=\frac{1}{3} G_{3}(\tau) G_{0}(\zeta)-G_{1}(\tau) G_{2}(\zeta) .
\end{gathered}
$$

The reader can find the complete set of the generalized eigenfunctions in [6]. The form of the generalized eigenfunctions indicates that $E^{4} \psi=0$ does not separate variables, but exhibits a kind of separation, which was called semiseparation. In Figure 7 and Figure 8, we draw streamlines in prolate geometry for the generalized eigenfunctions $\Omega_{1}(\tau, \zeta), \Omega_{2}(\tau, \zeta)$ of $E^{2}$.

\subsection{Separation and Semiseparation in Oblate Spheroidal Geometry}

Any point $\left(x_{1}, x_{2}, x_{3}\right)$ in the Cartesian coordinate system, is expressed using the oblate spheroid coordinates $(\lambda, \zeta, \varphi)$ where $\lambda \in \mathbb{R}, \zeta \in[-1,1]$ and $\alpha>0$ is the semifocal distance with

$$
\left(x_{1}, x_{2}, x_{3}\right)=\left(\alpha \sqrt{\lambda^{2}+1} \sqrt{1-\zeta^{2}} \cos (\varphi), \alpha \sqrt{\lambda^{2}+1} \sqrt{1-\zeta^{2}} \sin (\varphi), \alpha \lambda \zeta\right) .
$$

Stokes operator assumes the form

$$
E^{2}=\frac{1}{a^{2}\left(\lambda^{2}+\zeta^{2}\right)}\left[\left(1+\lambda^{2}\right) \frac{\partial^{2}}{\partial \lambda^{2}}+\left(1-\zeta^{2}\right) \frac{\partial^{2}}{\partial \zeta^{2}}\right] .
$$

Equation $E^{2} \psi=0$ separates variables and the eigenfunctions are

$$
G_{n}(i \lambda) G_{n}(\zeta), G_{n}(i \lambda) H_{n}(\zeta), H_{n}(i \lambda) G_{n}(\zeta), H_{n}(i \lambda) H_{n}(\zeta) .
$$

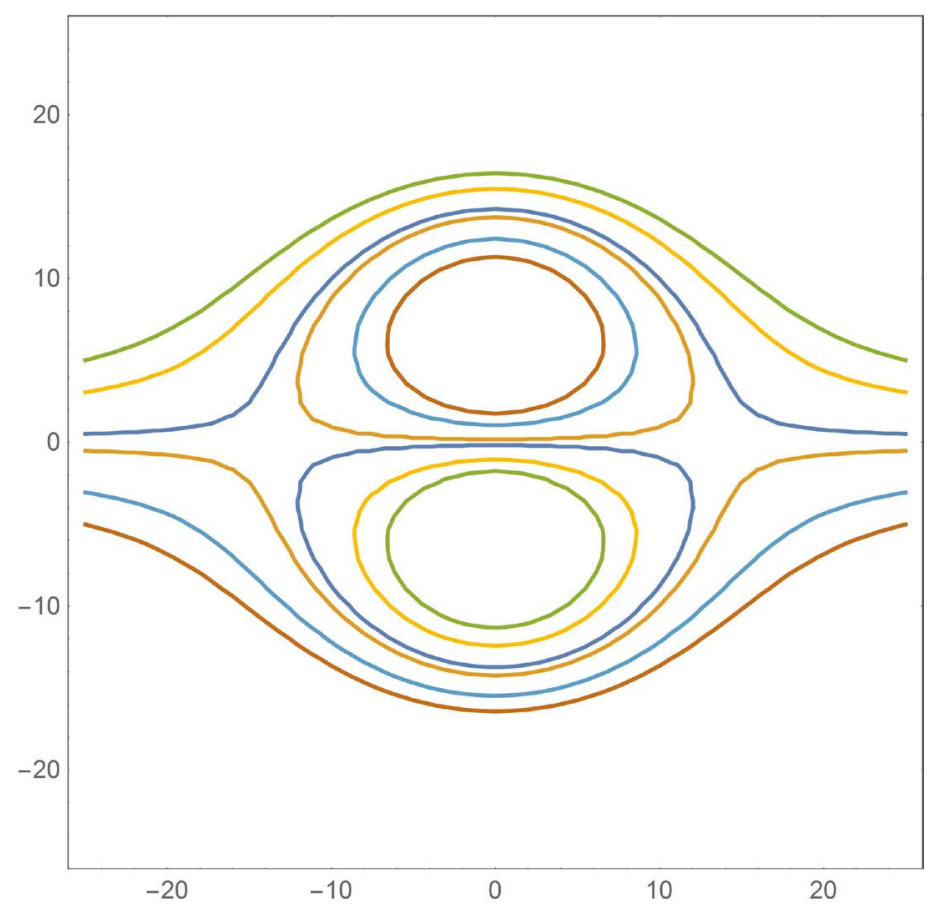

Figure 7. Streamlines for $-\frac{1}{3} G_{0}(\tau) G_{3}(\zeta)+G_{2}(\tau) G_{1}(\zeta)$ in prolate spheroid. 


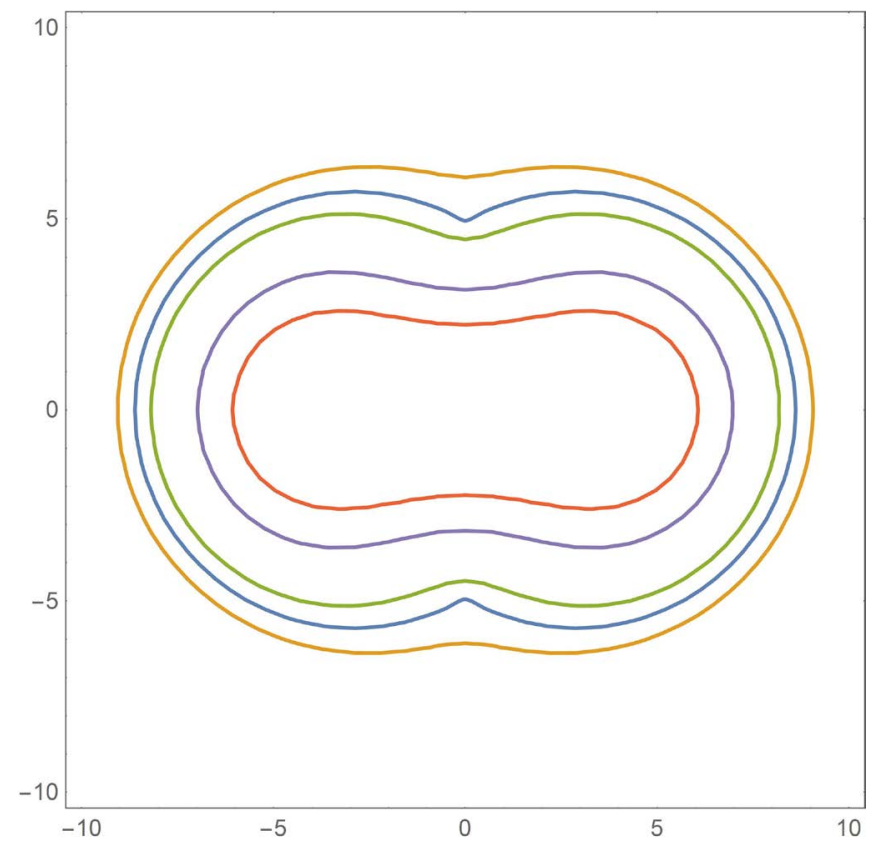

Figure 8. Streamlines for $\frac{1}{3} G_{3}(\tau) G_{0}(\zeta)-G_{1}(\tau) G_{2}(\zeta)$ in prolate spheroid.

The generalized eigenfunctions are given as products of Gegenbauer functions of mixed order, such as:

$a_{n}^{*}\left[G_{n-2}(i \lambda) G_{n}(\zeta)+G_{n}(i \lambda) G_{n-2}(\zeta)\right]+b_{n}^{*}\left[G_{n+2}(i \lambda) G_{n}(\zeta)+G_{n}(i \lambda) G_{n+2}(\zeta)\right], n \geq 4$.

These eigenfunctions indicate that $E^{4} \psi=0$ in the oblate geometry also semiseparates variables.

\subsection{R-Separation and R-Semiseparation in Inverted Prolate Spheroidal Geometry}

The inversion of convex geometrical objects with respect to a sphere with the same origin, creates interesting non-convex shapes, many of them resemble physical or biological entities. Their use in mathematical models and the analytical treatment of which dictates the "translation" of the problem at hand to the particular inverse coordinate system. Any point $\left(x_{1}, x_{2}, x_{3}\right)$ on the Cartesian coordinate system is defined in the inverted prolate spheroidal coordinates as

$$
\left(x_{1}, x_{2}, x_{3}\right)=\left(\frac{\sqrt{\tau^{2}-1} \sqrt{1-\zeta^{2}} \cos (\phi)}{c\left(\tau^{2}+\zeta^{2}-1\right)}, \frac{\sqrt{\tau^{2}-1} \sqrt{1-\zeta^{2}} \sin (\phi)}{c\left(\tau^{2}+\zeta^{2}-1\right)}, \frac{\tau \zeta}{c\left(\tau^{2}+\zeta^{2}-1\right)}\right),
$$

where $c>0$ is the semifocal distance and $\tau \geq 1, \zeta \in[-1,1], \phi \in[0,2 \pi)$. Stokes operator assumes the form

$$
\begin{aligned}
E^{2}= & \frac{c^{2}\left(\tau^{2}+\zeta^{2}-1\right)}{b^{4}\left(\tau^{2}-\zeta^{2}\right)}\left\{2 \tau\left(\tau^{2}-1\right) \frac{\partial}{\partial \tau}+\left(\tau^{2}-1\right)\left(\tau^{2}+\zeta^{2}-1\right) \frac{\partial^{2}}{\partial \tau^{2}}\right. \\
& \left.+2 \zeta\left(1-\zeta^{2}\right) \frac{\partial}{\partial \zeta}+\left(1-\zeta^{2}\right)\left(\tau^{2}+\zeta^{2}-1\right) \frac{\partial^{2}}{\partial \zeta^{2}}\right\} .
\end{aligned}
$$


Stokes equation $\mathrm{R}$-separates variables with $R(\tau, \zeta)$ being the Euclidean distance $r$ and the eigenfunctions [23] are

$$
\frac{1}{r} G_{n}(\tau) G_{n}(\zeta), \frac{1}{r} G_{n}(\tau) H_{n}(\zeta), \frac{1}{r} H_{n}(\tau) G_{n}(\zeta), \frac{1}{r} H_{n}(\tau) H_{n}(\zeta),
$$

where

$$
r=c \sqrt{\tau^{2}+\zeta^{2}-1}
$$

In Figure 9 and Figure 10, we depict sample streamlines in the inverted prolate geometry for the eigenfunctions $\frac{H_{3}(\tau) G_{3}(\zeta)}{\sqrt{\tau^{2}+\zeta^{2}-1}}, \frac{H_{3}(\tau) H_{3}(\zeta)}{\sqrt{\tau^{2}+\zeta^{2}-1}}$ that satisfy $E^{2} \psi=0$.

Moreover, the generalized eigenfunctions can not be obtained in closed form, but they can be calculated through recurrence relations [23]. Sample eigenfunctions are given below.

$$
\begin{gathered}
\Omega_{3}(\tau, \zeta)=\frac{G_{2}(\tau) H_{2}(\zeta)}{-2{\sqrt{\tau^{2}+\zeta^{2}-1}}^{3}}+\frac{G_{2}(\tau) G_{1}(\zeta)}{-3{\sqrt{\tau^{2}+\zeta^{2}-1}}^{3}} \\
\Omega_{4}(\tau, \zeta)=\frac{H_{3}(\tau) H_{3}(\zeta)}{-6{\sqrt{\tau^{2}+\zeta^{2}-1}}^{3}}+\frac{G_{0}(\tau) H_{3}(\zeta)}{9{\sqrt{\tau^{2}+\zeta^{2}-1}}^{3}}+\frac{H_{3}(\tau) G_{0}(\zeta)}{9{\sqrt{\tau^{2}+\zeta^{2}-1}}^{3}} .
\end{gathered}
$$

In Figure 11 and Figure 12, we present streamlines in the inverted prolate geometry for the generalized eigenfunctions $\Omega_{3}(\tau, \zeta), \Omega_{4}(\tau, \zeta)$ of $E^{2}$.

It has been proved that Stokes bistream equation, $E^{4} \psi=0$, R-semiseparates variables [23] [24], with $R$ being the third power of the Euclidean distance r, i.e. $r^{3}$.

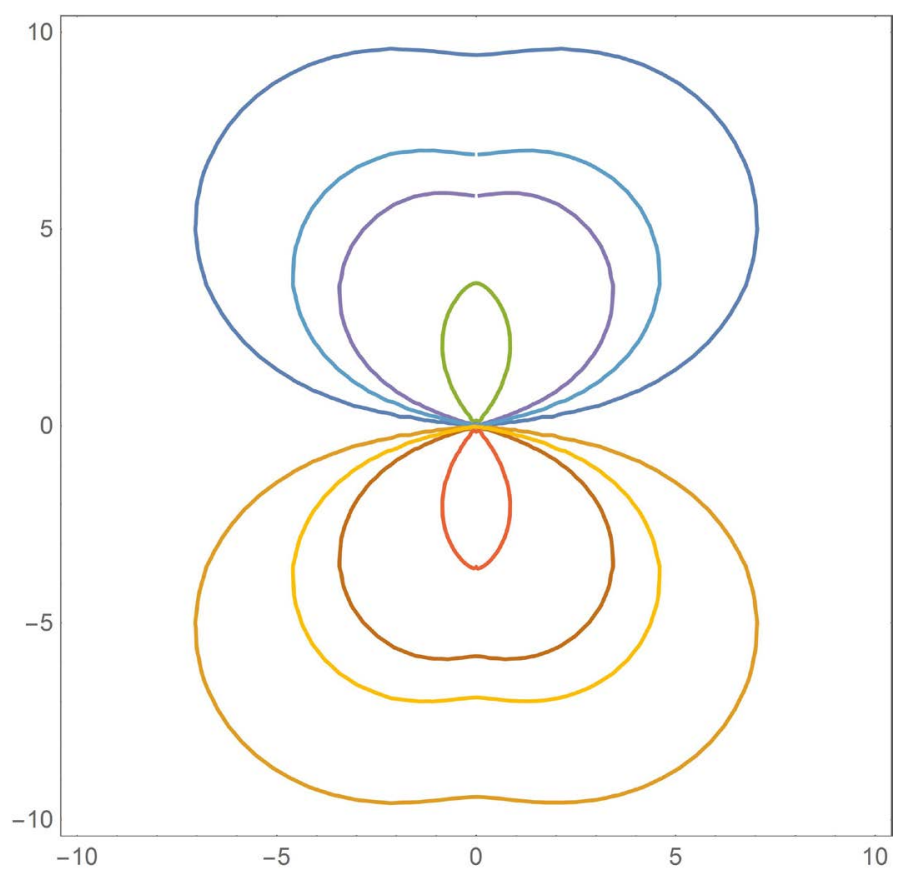

Figure 9. Streamlines for $\frac{H_{3}(\tau) G_{3}(\zeta)}{\sqrt{\tau^{2}+\zeta^{2}-1}}$ in inverted prolate geometry. 


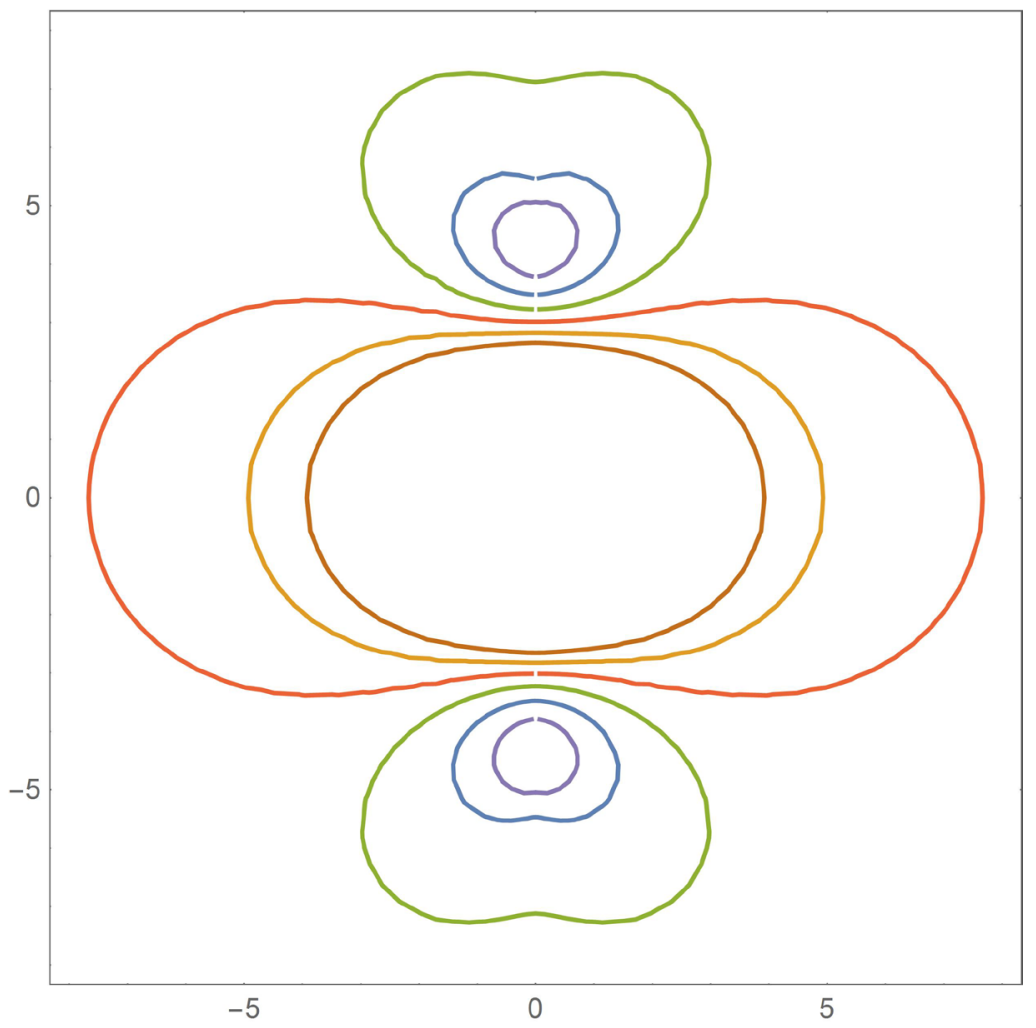

Figure 10. Streamlines for $\frac{H_{3}(\tau) H_{3}(\zeta)}{\sqrt{\tau^{2}+\zeta^{2}-1}}$ in inverted prolate geometry.

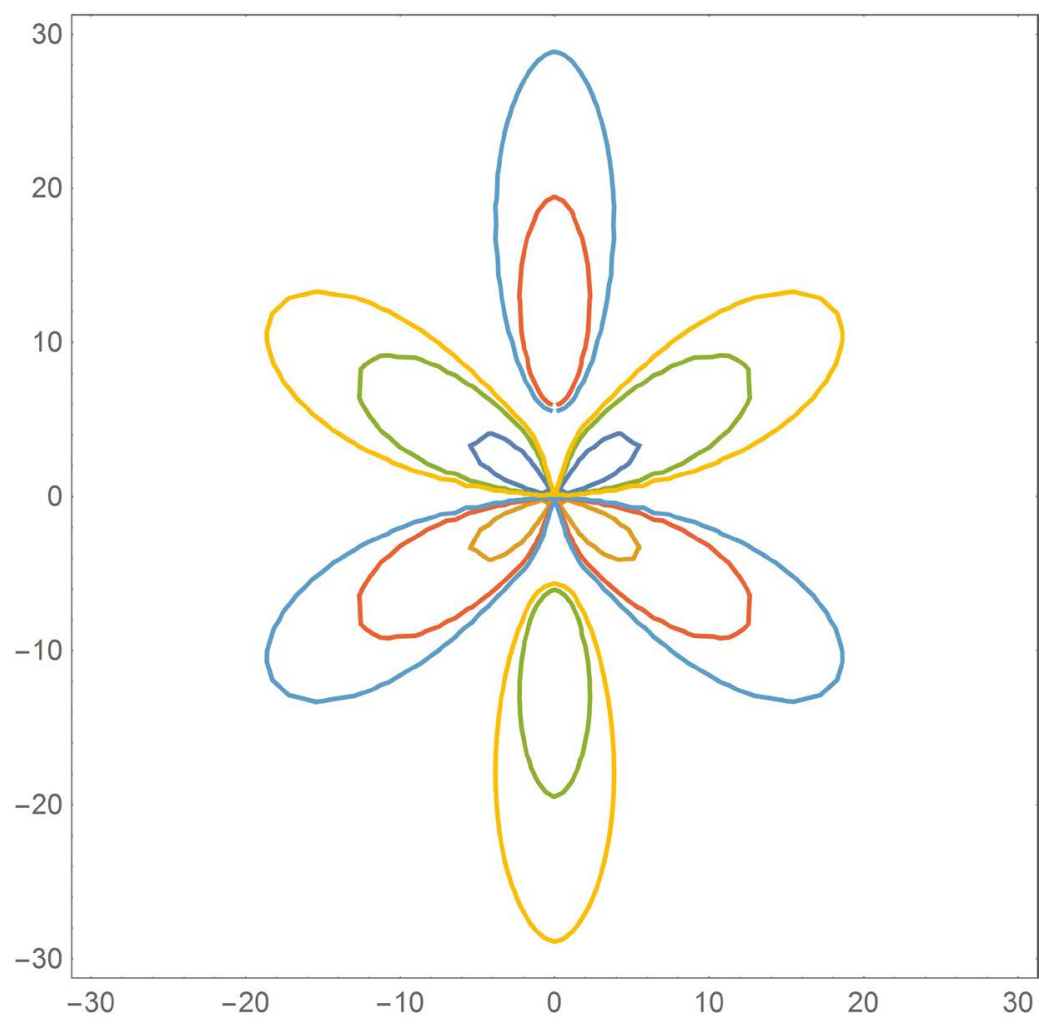

Figure 11. Streamlines for $\Omega_{1}(\tau, \zeta)$ in inverted prolate geometry. 


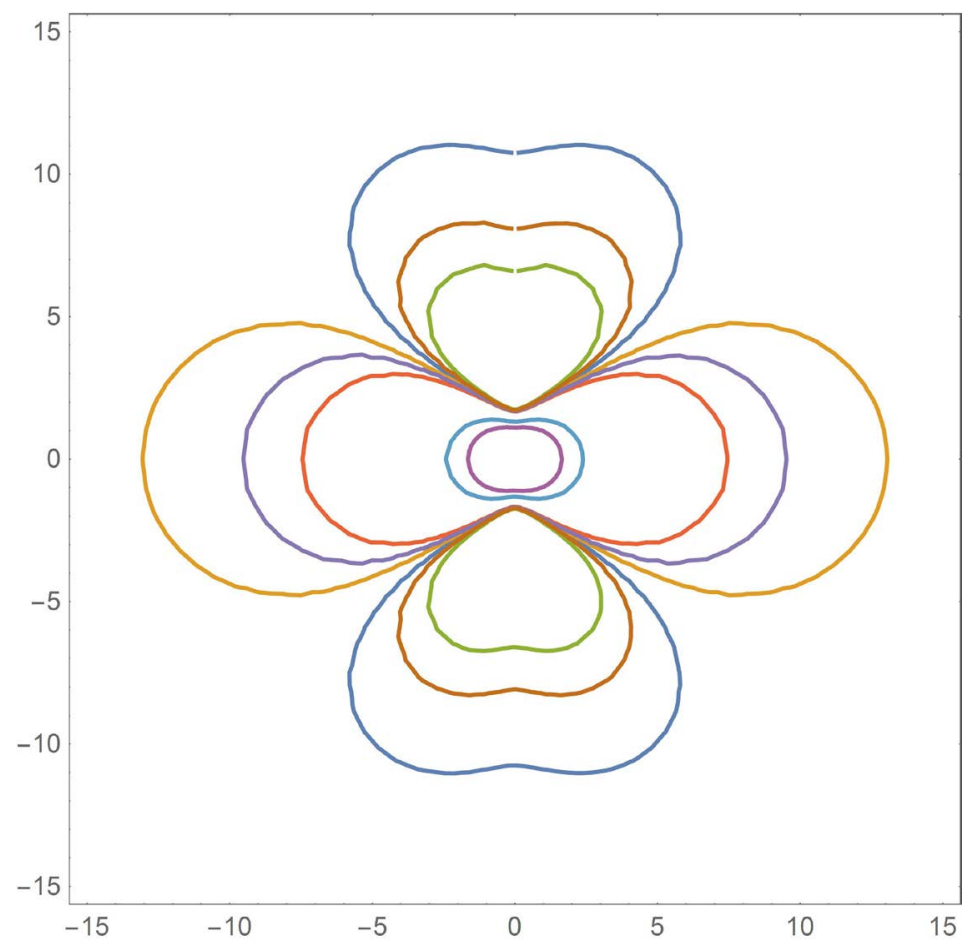

Figure 12. Streamlines for $\Omega_{1}(\tau, \zeta)$ in inverted prolate geometry.

\subsection{R-Separation and R-Semiseparation Inverted Oblate Spheroidal Geometry}

Any point $\left(x_{1}, x_{2}, x_{3}\right)$ on the Cartesian coordinate system is defined in the inverted oblate spheroidal coordinates as

$$
\left(x_{1}, x_{2}, x_{3}\right)=\left(\frac{\sqrt{\lambda^{2}+1} \sqrt{1-\zeta^{2}} \cos (\phi)}{a\left(\lambda^{2}-\zeta^{2}+1\right)}, \frac{\sqrt{\lambda^{2}+1} \sqrt{1-\zeta^{2}} \sin (\phi)}{a\left(\lambda^{2}-\zeta^{2}+1\right)}, \frac{\lambda \zeta}{a\left(\lambda^{2}-\zeta^{2}+1\right)}\right),
$$

where $a>0$ is the semifocal distance and $\lambda \in \mathbb{R}, \zeta \in[-1,1], \phi \in[0,2 \pi)$.

Stokes operator is

$$
\begin{aligned}
E^{2}= & \frac{a^{2}\left(\lambda^{2}-\zeta^{2}+1\right)}{b^{4}\left(\lambda^{2}+\zeta^{2}\right)}\left\{2 \lambda\left(\lambda^{2}+1\right) \frac{\partial}{\partial \lambda}+\left(\lambda^{2}+1\right)\left(\lambda^{2}-\zeta^{2}+1\right) \frac{\partial^{2}}{\partial \lambda^{2}}\right. \\
& \left.\left.-2 \zeta\left(1-\zeta^{2}\right) \frac{\partial}{\partial \zeta}+\left(1-\zeta^{2}\right)\right)\left(\lambda^{2}-\zeta^{2}+1\right) \frac{\partial^{2}}{\partial \zeta^{2}}\right\} .
\end{aligned}
$$

Stokes equation R-separates variables and the eigenfunctions [25] are

$$
\frac{1}{r} G_{n}(i \lambda) G_{n}(\zeta), \frac{1}{r} G_{n}(i \lambda) H_{n}(\zeta), \frac{1}{r} H_{n}(i \lambda) G_{n}(\zeta), \frac{1}{r} H_{n}(i \lambda) H_{n}(\zeta),
$$

with $R(\lambda, \zeta)$ being the Euclidean distance $r=a \sqrt{\lambda^{2}-\zeta^{2}+1}$.

Moreover, as in the inverted prolate spheroidal case, the generalized eigenfunction can not be derived in closed form, but they can be calculated through recurrence relations [25]. These eigenfunctions are sum of products of Gegenbauer functions of different order, multiplied by the function $r^{-3}$ and of the form given below 


$$
-\frac{5 G_{2}(i \lambda) H_{2}(\zeta)}{8{\sqrt{\lambda^{2}-\zeta^{2}+1}}^{3}}-\frac{25 G_{2}(i \lambda) G_{1}(\zeta)}{72{\sqrt{\lambda^{2}-\zeta^{2}+1}}^{3}} .
$$

It has been proved [25] that Stokes bistream equation $\mathrm{R}$-semiseparates variables [25], with $R$ being the Euclidean distance on the third, i.e. $a^{3}{\sqrt{\lambda^{2}-\zeta^{2}+1}}^{3}$.

\section{Separability of Equation $E^{2} \psi=0$ in Other Axisymmetric Geometries}

Next, we provide results in other than the spherical and the spheroidal systems of coordinates.

\subsection{R-Separation in Bispherical Geometry}

In bispherical coordinate system $(\eta, \theta, \varphi)$ any point $\left(x_{1}, x_{2}, x_{3}\right)$ in the Cartesian coordinates system [1] is expressed as

$$
\left(x_{1}, x_{2}, x_{3}\right)=\left(\frac{\alpha \sin (\theta) \cos (\varphi)}{\cosh (\eta)-\cos (\theta)}, \frac{\alpha \sin (\theta) \sin (\varphi)}{\cosh (\eta)-\cos (\theta)}, \frac{\alpha \sinh (\eta)}{\cosh (\eta)-\cos (\theta)}\right),
$$

where $a>0, \eta \in \mathbb{R}, \theta \in[0, \pi)$, while Stokes operator assumes the form

$$
\begin{aligned}
E^{2}= & \frac{[\cosh (\eta)-\cos (\theta)]^{2}}{a^{2}}\left\{\frac{\partial^{2}}{\partial \eta^{2}}+\frac{\sinh (\eta)}{\cosh (\eta)-\cos (\theta)} \frac{\partial}{\partial \eta}\right. \\
& \left.+\frac{\csc (\theta)-\cosh (\eta) \cot (\theta)}{\cosh (\eta)-\cos (\theta)} \frac{\partial}{\partial \theta}+\frac{\partial^{2}}{\partial \theta^{2}}\right\} .
\end{aligned}
$$

Equation $E^{2} \psi=0 \quad \mathrm{R}$-separates variables [22] and the eigenfunctions are

$$
\begin{aligned}
& \frac{1}{\cosh (\eta)-\cos (\theta)} \cosh (n \eta) G_{n+1 / 2}(\cos (\theta)), \\
& \frac{1}{\cosh (\eta)-\cos (\theta)} \sinh (n \eta) G_{n+1 / 2}(\cos (\theta)), \\
& \frac{1}{\cosh (\eta)-\cos (\theta)} \cosh (n \eta) H_{n+1 / 2}(\cos (\theta)), \\
& \frac{1}{\cosh (\eta)-\cos (\theta)} \sinh (n \eta) H_{n+1 / 2}(\cos (\theta)),
\end{aligned}
$$

with $R(\eta, \theta)=\cosh (\eta)-\cos (\theta)$.

In Figure 13 and Figure 14, we present sample streamlines in bispherical geometry for the eigenfunctions $\frac{\sinh (2 \eta) G_{5 / 2}(\cos (\theta))}{\cosh (\eta)-\cos (\theta)}, \frac{\sinh (3 \eta) H_{7 / 2}(\cos (\theta))}{\cosh (\eta)-\cos (\theta)}$ of $E^{2}$.

\subsection{R-Separation in Toroidal Geometry}

In toroidal coordinate system $(\eta, \theta, \varphi)$ any point $\left(x_{1}, x_{2}, x_{3}\right)$ in the Cartesian coordinates system [1] is expressed with

$$
\left(x_{1}, x_{2}, x_{3}\right)=\left(\frac{\alpha \sinh (\eta) \cos (\varphi)}{\cosh (\eta)-\cos (\theta)}, \frac{\alpha \sinh (\theta) \sin (\varphi)}{\cosh (\eta)-\cos (\theta)}, \frac{\alpha \sin (\theta)}{\cosh (\eta)-\cos (\theta)}\right),
$$




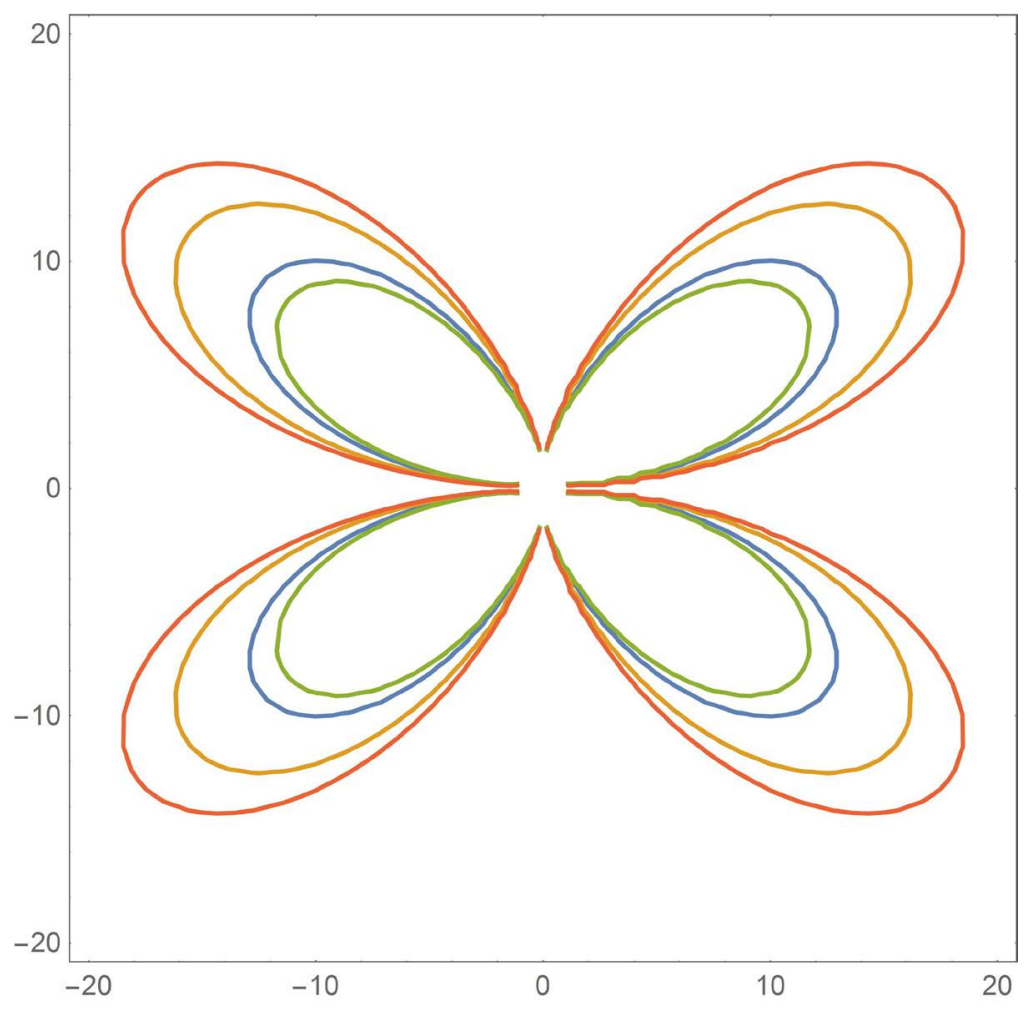

Figure 13. Streamlines for $\frac{\sinh (2 \eta) G_{5 / 2}(\cos (\theta))}{\cosh (\eta)-\cos (\theta)}$ in bispherical geometry.

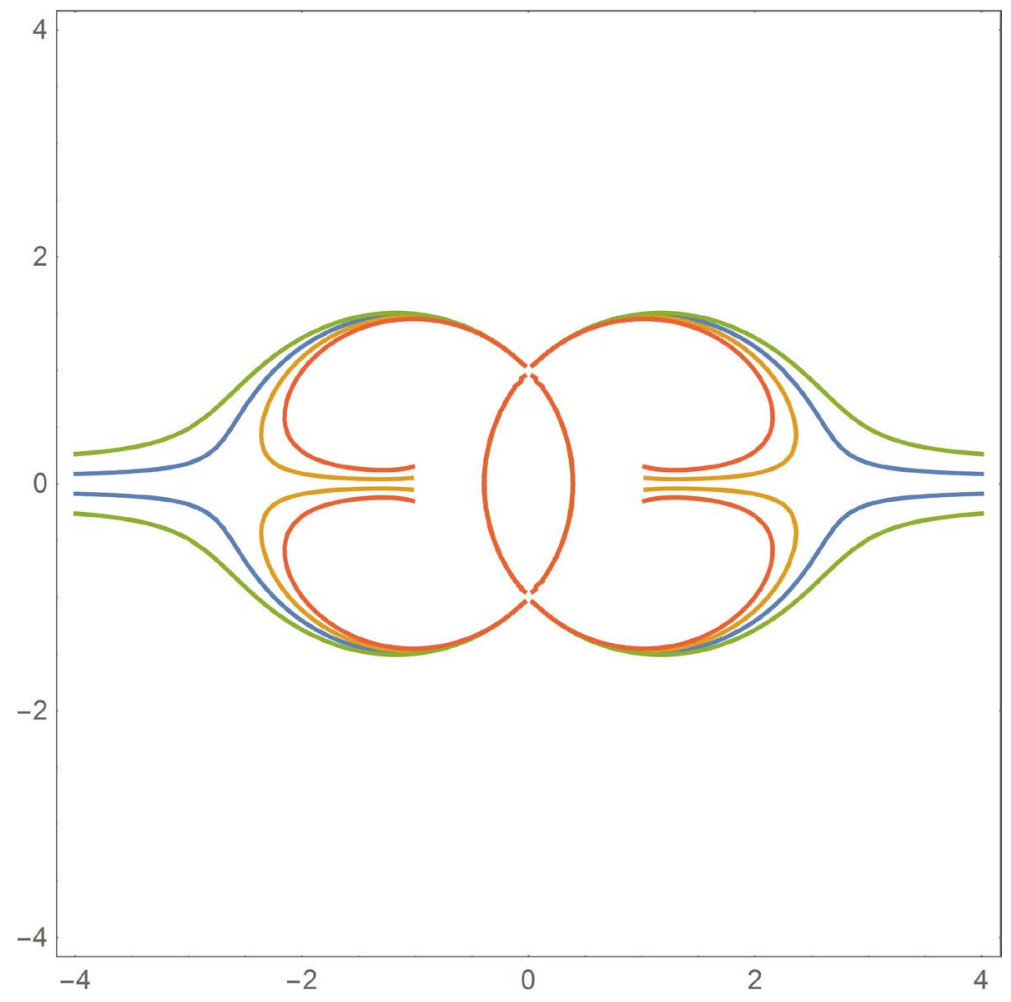

Figure 14. Streamlines for $\frac{\sinh (3 \eta) H_{7 / 2}(\cos (\theta))}{\cosh (\eta)-\cos (\theta)}$ in bispherical geometry. 
where $a>0, \eta \geq 0, \theta \in(-\pi, \pi]$ and Stokes operator assumes the form

$$
\begin{aligned}
E^{2}= & \frac{[\cosh (\eta)-\cos (\theta)]^{2}}{a^{2}}\left\{\frac{\partial^{2}}{\partial \eta^{2}}+\frac{\cos (\theta) \cosh (\eta)-1}{\sinh (\eta)[\cosh (\eta)-\cos (\theta)]} \frac{\partial}{\partial \eta}\right. \\
& \left.+\frac{\sin (\theta)}{\cosh (\eta)-\cos (\theta)} \frac{\partial}{\partial \theta}+\frac{\partial^{2}}{\partial \theta^{2}}\right\} .
\end{aligned}
$$

Equation $E^{2} \psi=0 \quad \mathrm{R}$-separates variables [22] and the eigenfunctions are

$$
\begin{aligned}
& \frac{1}{\cosh (\eta)-\cos (\theta)} G_{n+1 / 2}(\cosh (\eta)) \cos (n \theta), \\
& \frac{1}{\cosh (\eta)-\cos (\theta)} G_{n+1 / 2}(\cosh (\eta)) \sin (n \theta), \\
& \frac{1}{\cosh (\eta)-\cos (\theta)} H_{n+1 / 2}(\cosh (\eta)) \cos (n \theta), \\
& \frac{1}{\cosh (\eta)-\cos (\theta)} H_{n+1 / 2}(\cosh (\eta)) \sin (n \theta),
\end{aligned}
$$

with $R(\eta, \theta)=\cosh (\eta)-\cos (\theta)$.

In Figure 15 and Figure 16, we present sample streamlines in toroidal geometry for the eigenfunctions $\frac{G_{5 / 2}(\cosh (\eta)) \sin (2 \theta)}{\cosh (\eta)-\cos (\theta)}, \frac{G_{7 / 2}(\cosh (\eta)) \sin (3 \theta)}{\cosh (\eta)-\cos (\theta)}$ that satisfy $E^{2} \psi=0$.

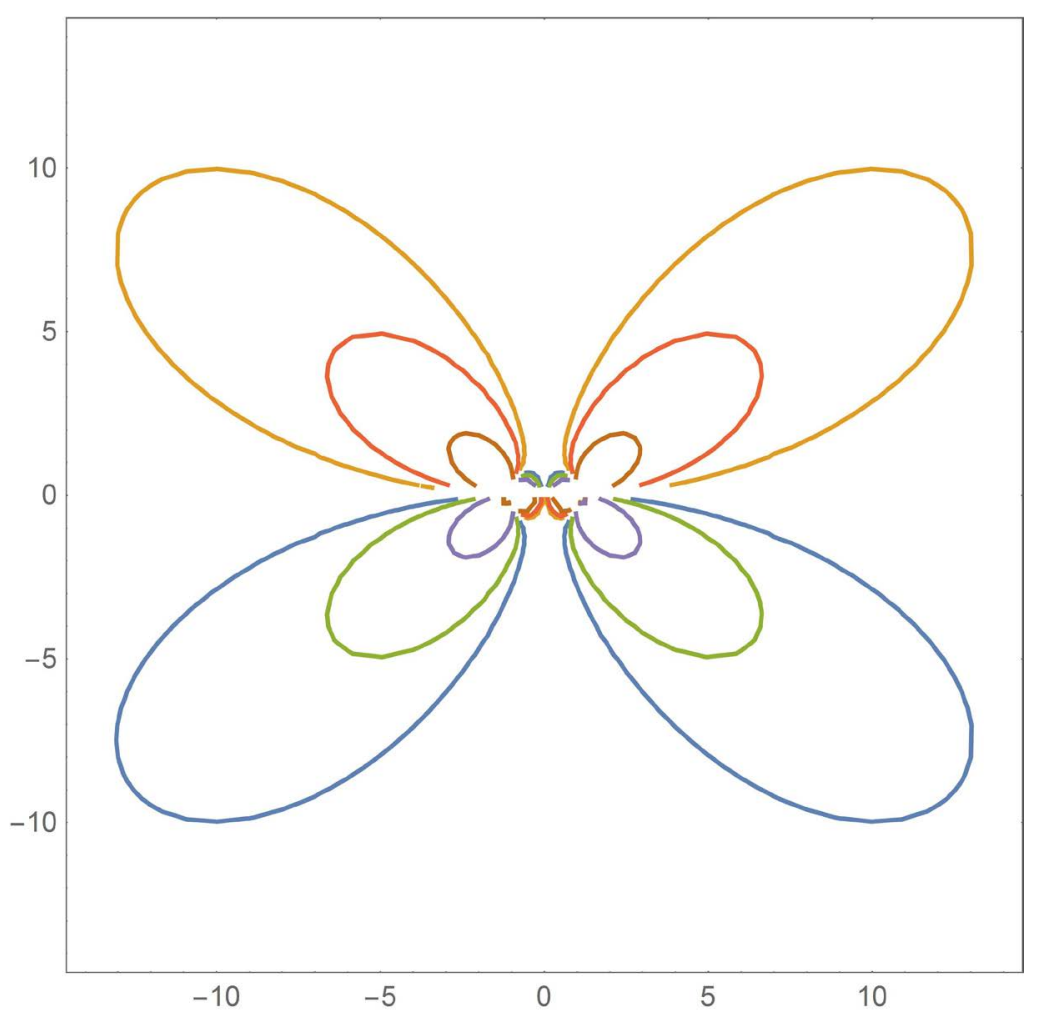

Figure 15. Streamlines for $\frac{G_{5 / 2}(\cosh (\eta)) \sin (2 \theta)}{\cosh (\eta)-\cos (\theta)}$ in toroidal geometry. 


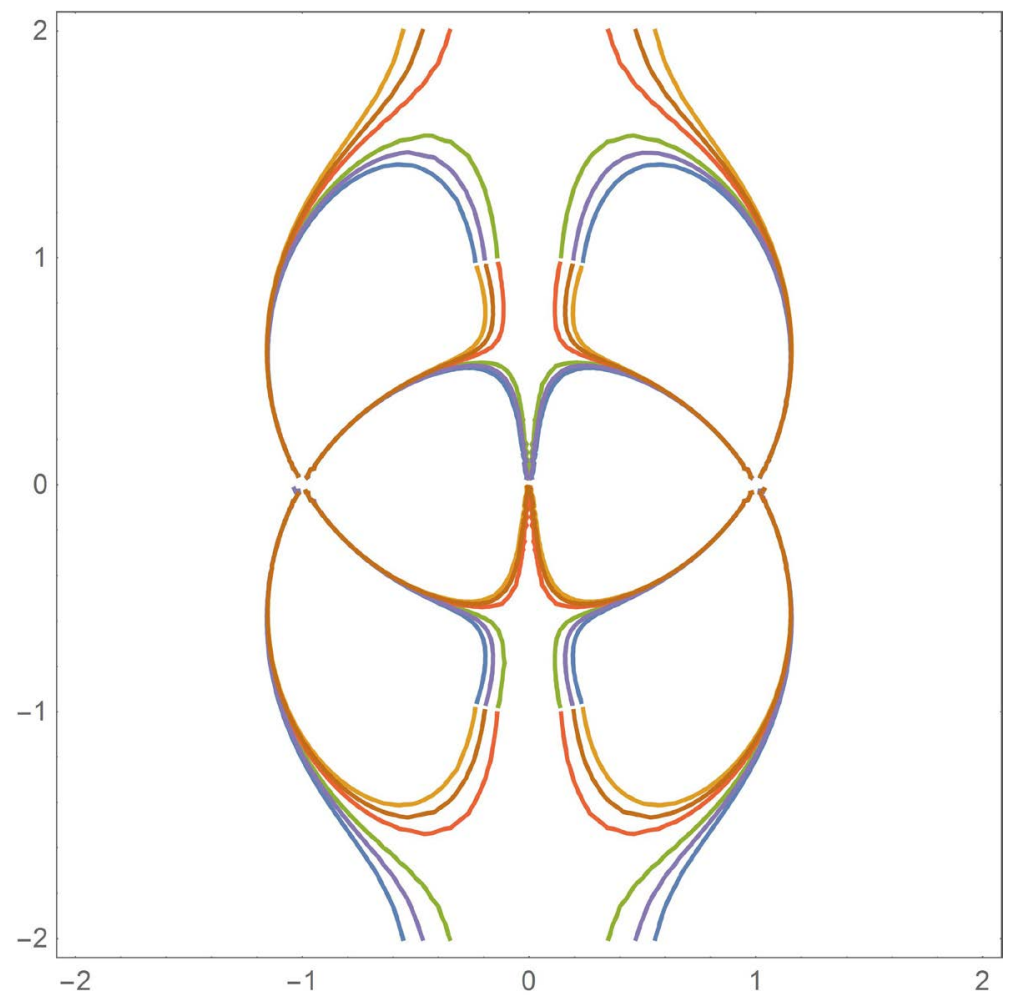

Figure 16. Streamlines for $\frac{G_{7 / 2}(\cosh (\eta)) \sin (3 \theta)}{\cosh (\eta)-\cos (\theta)}$ in toroidal geometry.

\subsection{Separation in Parabolic Geometry}

In parabolic coordinate system $(\mu, v, \varphi)$ any point $\left(x_{1}, x_{2}, x_{3}\right)$ in the Cartesian coordinates system [1] is expressed as

$$
\left(x_{1}, x_{2}, x_{3}\right)=\left(\mu \nu \cos (\varphi), \mu v \sin (\varphi), \frac{\mu^{2}-v^{2}}{2}\right) \text {, }
$$

where $\mu, v \geq 0$, while Stokes operator assumes the form

$$
E^{2}=\frac{1}{\mu^{2}+v^{2}}\left(\frac{\partial^{2}}{\partial \mu^{2}}-\frac{1}{\mu} \frac{\partial}{\partial \mu}-\frac{1}{v} \frac{\partial}{\partial v}+\frac{\partial^{2}}{\partial v^{2}}\right) .
$$

Equation $E^{2} \psi=0$ separates variables [30] and the eigenfunctions are $\mu v I_{1}(n \mu) J_{1}(n v), \mu v I_{1}(n \mu) Y_{1}(n v), \mu v K_{1}(n \mu) J_{1}(n v), \mu v K_{1}(n \mu) Y_{1}(n v)$,

where $J_{1}, Y_{1}$ are Bessel functions of the first order and first and second kind respectively and $I_{1}, K_{1}$ are modified Bessel functions of the first order and first and second kind respectively [29]. In Figure 17 and Figure 18, we depict streamlines in parabolic geometry for the eigenfunctions $\mu v I_{1}(2 \mu) Y_{1}(2 v), \mu v K_{1}(3 \mu) Y_{1}(3 v)$ of $E^{2}$.

\subsection{R-Separation in Tangent Sphere Geometry}

In tangent sphere coordinates system $(\mu, v, \varphi)$ any point $\left(x_{1}, x_{2}, x_{3}\right)$ in the Cartesian coordinate system [1] is expressed as 


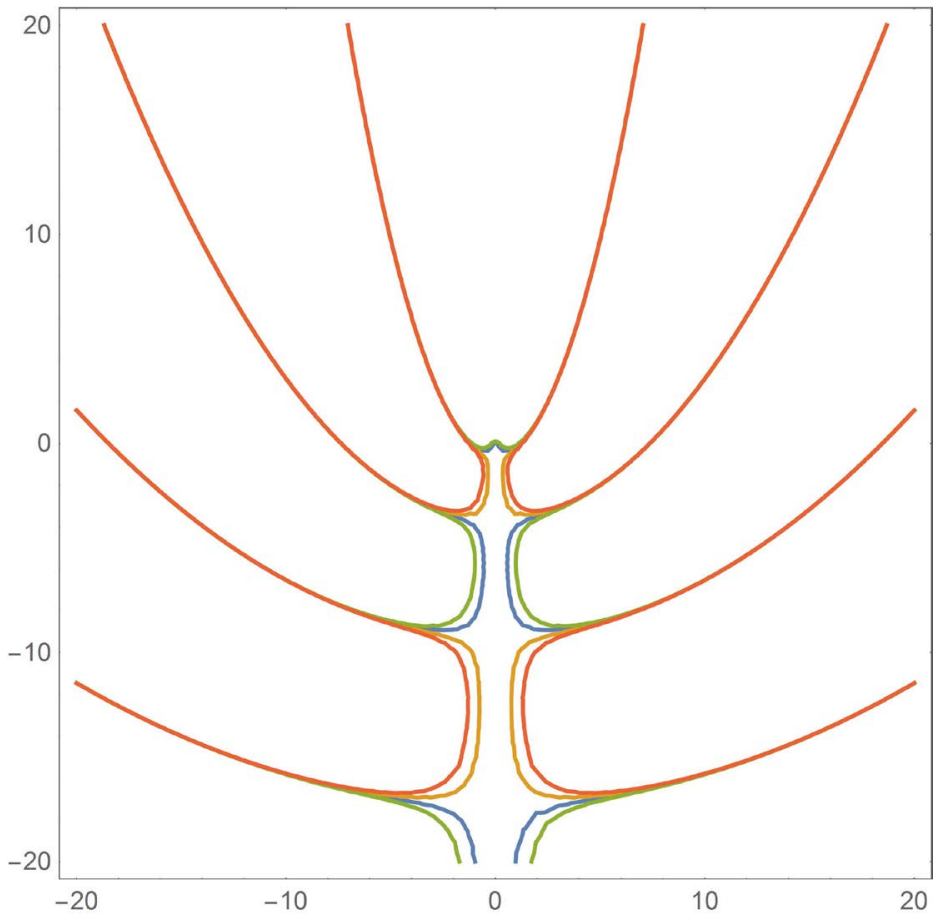

Figure 17. Streamlines for $\mu \nu I_{1}(2 \mu) Y_{1}(2 v)$ in parabolic geometry.

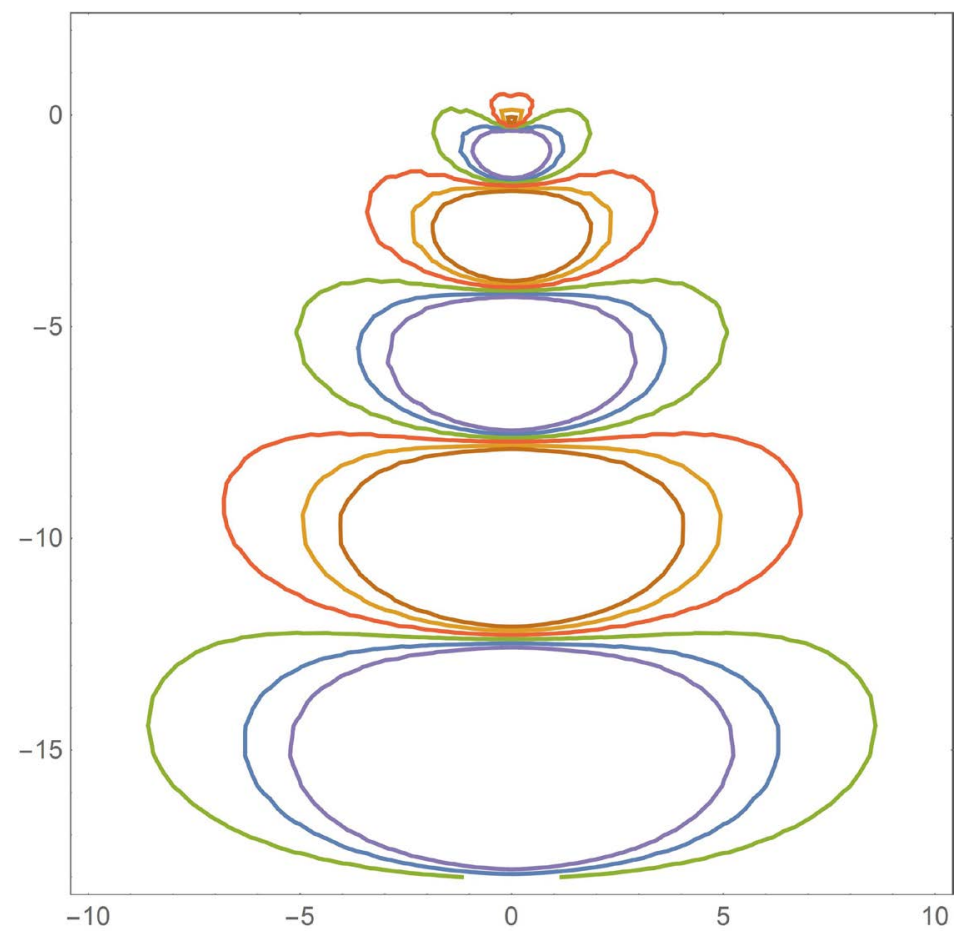

Figure 18. Streamlines for $\mu \nu K_{1}(3 \mu) Y_{1}(3 v)$ in parabolic geometry.

$$
\left(x_{1}, x_{2}, x_{3}\right)=\left(\frac{\mu \cos (\varphi)}{\mu^{2}+v^{2}}, \frac{\mu \sin (\varphi)}{\mu^{2}+v^{2}}, \frac{v}{\mu^{2}+v^{2}}\right),
$$

where $\mu>0, v \in \mathbb{R}$, while Stokes operator [30] assumes the form 


$$
E^{2}=\left(\mu^{2}+v^{2}\right)^{2}\left[\frac{\partial^{2}}{\partial \mu^{2}}+\frac{\mu^{2}-v^{2}}{\mu\left(\mu^{2}+v^{2}\right)} \frac{\partial}{\partial \mu}+\frac{2 v}{\mu^{2}+v^{2}} \frac{\partial}{\partial v}+\frac{\partial^{2}}{\partial v^{2}}\right]
$$

Stokes equation $E^{2} \psi(\mu, v)=0$ R-separates variables [30] and the eigenfunctions are

$$
\mu r I_{1}(n \mu) \cos (n v), \mu r I_{1}(n \mu) \sin (n v), \mu r K_{1}(n \mu) \cos (n v), \mu r K_{1}(n \mu) \sin (n v),(55
$$

with $R(\mu, v)$ being the inverse of the Euclidean distance $r=\frac{1}{\sqrt{\mu^{2}+v^{2}}}$. In Figure 19 and Figure 20, we depict streamlines in tangent sphere geometry for the eigenfunctions $\mu r K_{1}(3 \mu) \cos (3 v), \mu r K_{1}(3 \mu) \sin (3 v)$ that satisfy $E^{2} \psi=0$.

\subsection{R-Separation in Cardioid Geometry}

In cardioid coordinate system $(\mu, v, \varphi)$ any point $\left(x_{1}, x_{2}, x_{3}\right)$ in the Cartesian coordinates system is expressed as

$$
\left(x_{1}, x_{2}, x_{3}\right)=\left(\frac{\mu v \cos (\varphi)}{\left(\mu^{2}+v^{2}\right)^{2}}, \frac{\mu v \sin (\varphi)}{\left(\mu^{2}+v^{2}\right)^{2}}, \frac{\mu^{2}-v^{2}}{2\left(\mu^{2}+v^{2}\right)^{2}}\right),
$$

where $\mu, v \geq 0$, while Stokes operator assumes the form

$$
E^{2}=\left(\mu^{2}+v^{2}\right)^{3}\left[\frac{\partial^{2}}{\partial \mu^{2}}+\frac{3 \mu^{2}-v^{2}}{\mu\left(\mu^{2}+v^{2}\right)} \frac{\partial}{\partial \mu}+\frac{3 v^{2}-\mu^{2}}{v\left(\mu^{2}+v^{2}\right)} \frac{\partial}{\partial v}+\frac{\partial^{2}}{\partial v^{2}}\right] .
$$

Equation $E^{2} \psi(\mu, v)=0$ R-separates variables and the corresponding eigenfunctions are

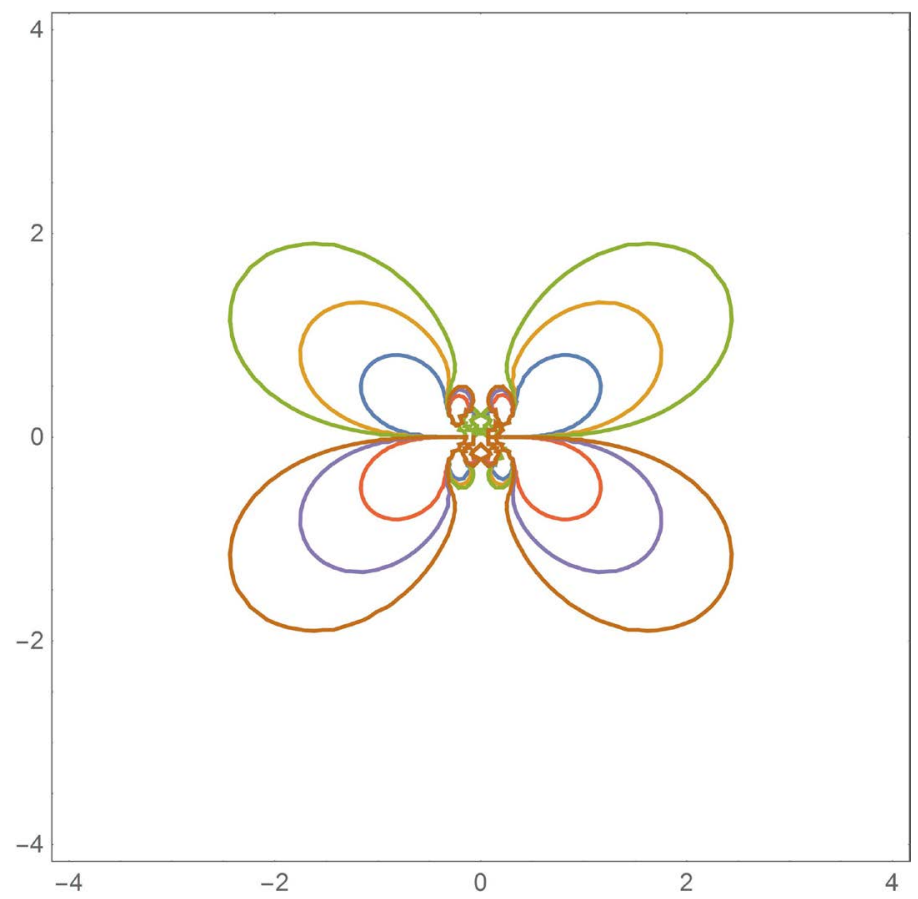

Figure 19. Streamlines for $\mu r K_{1}(3 \mu) \cos (3 v)$ in tangent sphere geometry. 


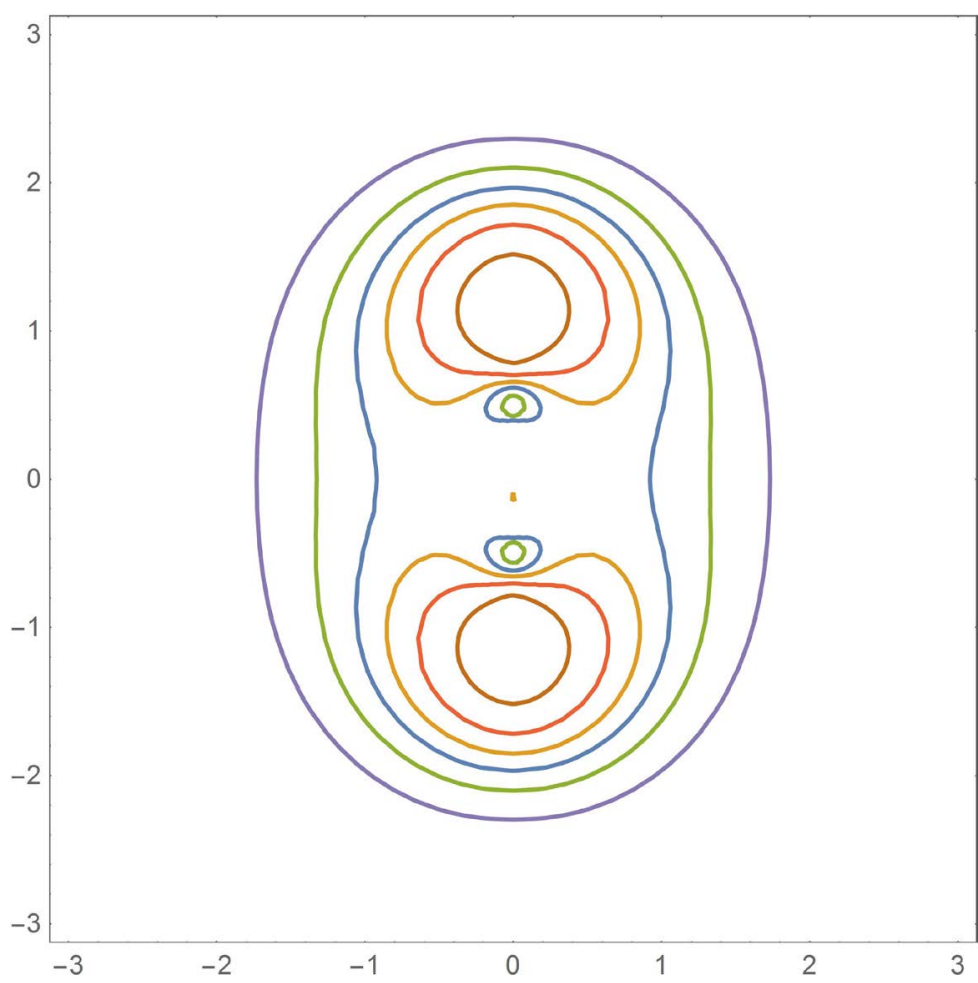

Figure 20. Streamlines for $\mu r K_{1}(3 \mu) \sin (3 v)$ in tangent sphere geometry.

$\mu v r I_{1}(n \mu) J_{1}(n v), \mu v r I_{1}(n \mu) Y_{1}(n v), \mu v r K_{1}(n \mu) J_{1}(n v), \mu v r K_{1}(n \mu) Y_{1}(n v)$.

with $R(\mu, v)$ being the inverse of the Euclidean distance $r=\frac{1}{\sqrt{\mu^{2}+v^{2}}}$. In Figure 21 and Figure 22, we draw sample streamlines in cardioid geometry for the eigenfunctions $\mu v r J_{1}(3 \mu) Y_{1}(3 v), \mu v r K_{1}(3 \mu) Y_{1}(3 v)$ of $E^{2}$.

\section{Applications in Biology}

\subsection{Relative Motion of Blood's Plasma Flow Past a Red Blood Cell}

Human's blood is a suspension of red blood cells (RBCs), white blood cells and the platelets within blood's plasma, which can be regarded as an incompressible Newtonian fluid. Blood's plasma is about $55 \%$ of the vessel volume, while the RBCs occupy about $43 \%$, leaving about $2 \%$ for white blood cells and the platelets, which proves the importance of the relative motion of blood's plasma past red blood cells. The physical characteristics of blood permit us to model the flow as axisymmetric Stokes flow around an inverted prolate spheroid which describes the RBC. We consider a uniform velocity $U$ parallel to $x_{3}$ axis in the negative direction and a stationary, isolated inverted prolate spheroid (Figure 23). The size of the RBC enables us to assume that the fluid extends to infinity [8].

The problem at hand is defined using the following Equation (59) through (62)

$$
E^{\prime 4} \psi_{a}\left(r^{\prime}\right)=0, r^{\prime} \in V^{\prime},
$$




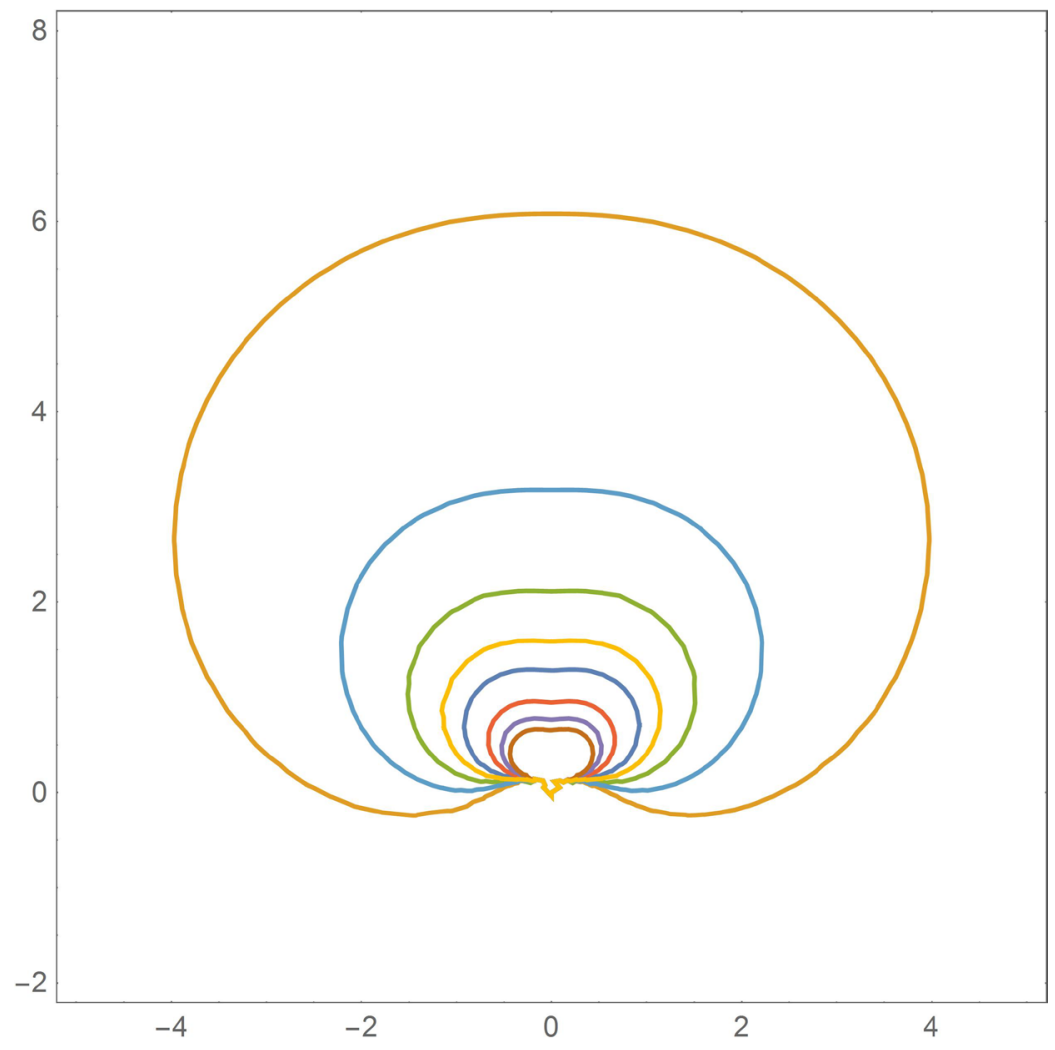

Figure 21. Streamlines for $\mu v r J_{1}(3 \mu) Y_{1}(3 v)$ in cardioid geometry.

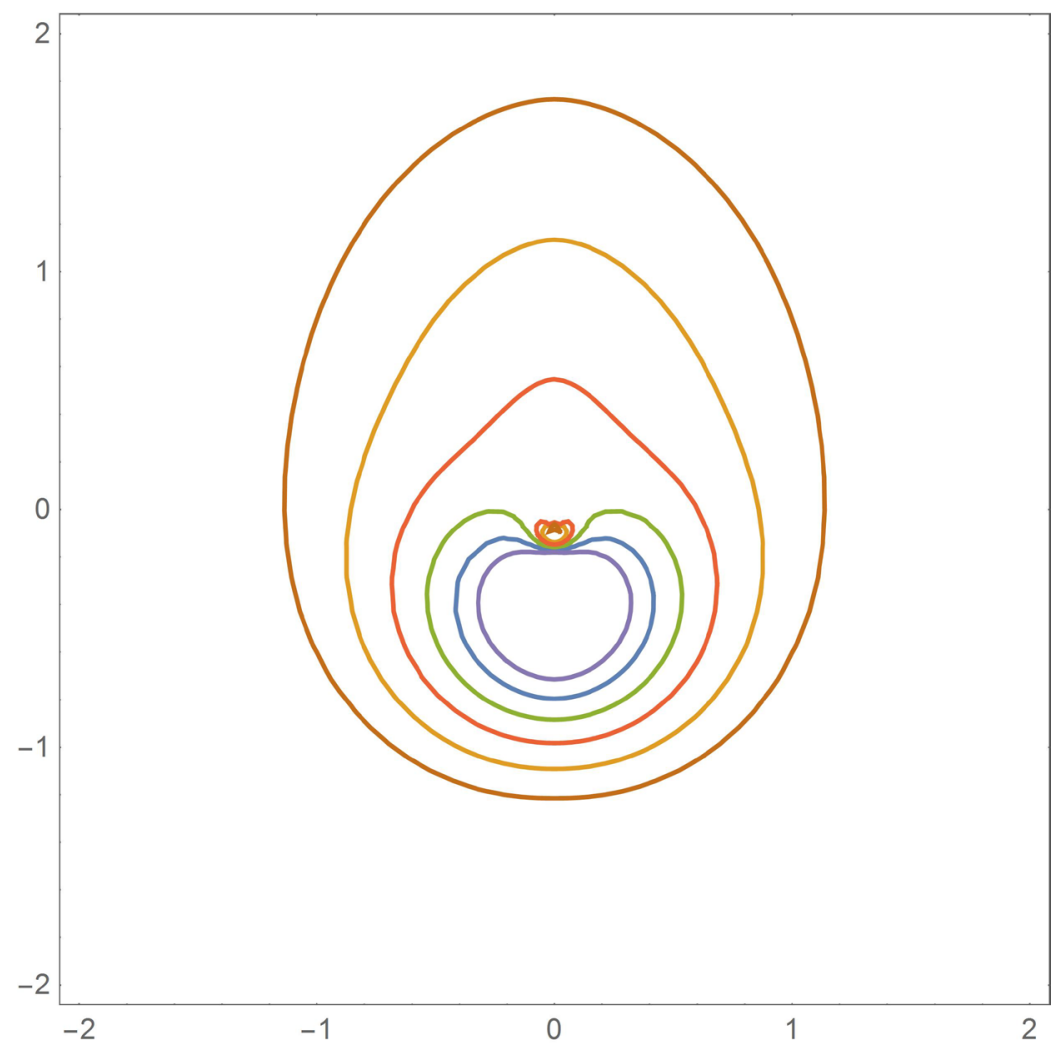

Figure 22. Streamlines for $\mu v r K_{1}(3 \mu) Y_{1}(3 v)$ in cardioid geometry. 


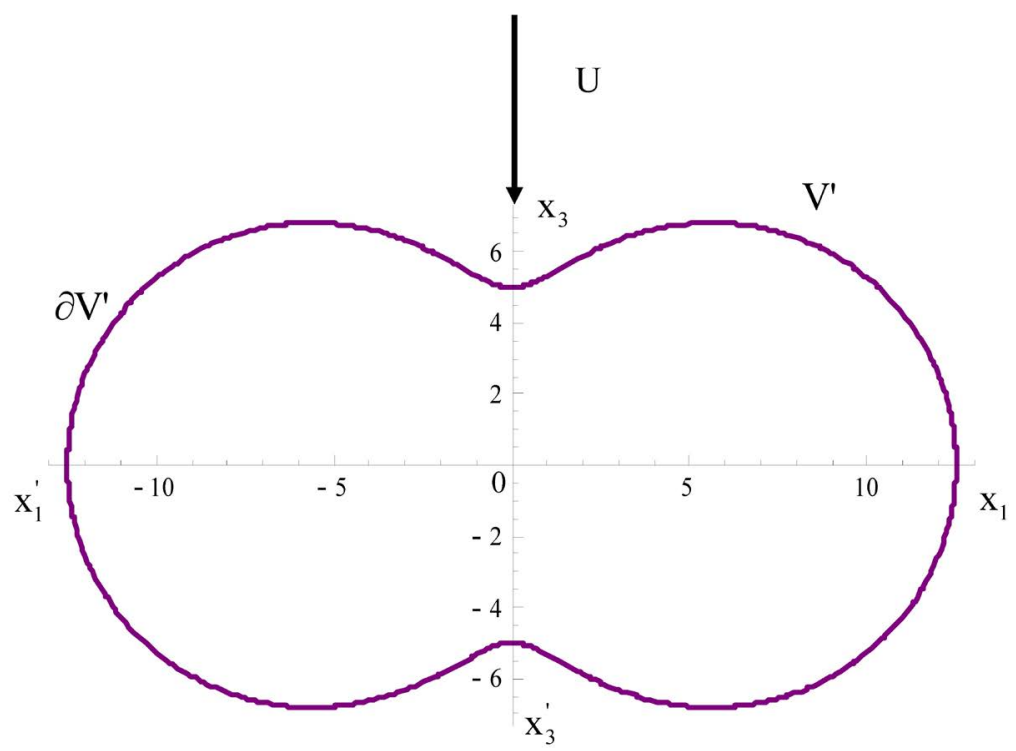

Figure 23. Blood's plasma flow past a RBC.

$$
\begin{gathered}
\psi_{a}\left(r^{\prime}\right)=0, r^{\prime} \in \partial V^{\prime}, \\
\frac{\partial \psi_{a}\left(r^{\prime}\right)}{\partial n}=0, r^{\prime} \in \partial V^{\prime}, \\
\psi_{a} \rightarrow \frac{1}{2} \varpi^{\prime 2} U, r^{\prime} \rightarrow+\infty,
\end{gathered}
$$

where $\psi_{a}\left(r^{\prime}\right)$ is the stream function, (59) denotes the Stokes equation, (60) defines the no slip condition on the surface of the RBC, while (61) expresses the impenetrability of the RBC and (62) denotes that the flow extends to infinity.

In order to solve the problem at hand, we employ the inverted prolate spheroid geometry using the variables $(\tau, \zeta, \phi)$ and we apply the boundary conditions having derive first new formulas for the Gegenbauer functions. This procedure results four equations with five unknown constants in each case. The final expression was derived using the fact that a prolate spheroid degenerates to a sphere when the semifocal distance tents to zero. The obtained stream function [8] is

$$
\psi_{a}(\tau, \zeta)=\frac{b^{3}}{c^{3}{\sqrt{\tau^{2}+\zeta^{2}-1}}^{3}} \sum_{n=1}^{+\infty} g_{2 n}(\tau) G_{2 n}(\zeta),
$$

where

$$
\begin{aligned}
g_{2}(\tau)=A_{2} G_{2}(\tau) & -\frac{9 b c U}{5} H_{2}(\tau)-b c U G_{1}(\tau)+E_{2} G_{4}(\tau)-\frac{b c U}{5} H_{4}(\tau) \\
g_{2 n}(\tau)= & A_{2 n} G_{2 n}(\tau)+\frac{b c U}{2}\left(-w_{n-1} e_{n-1}^{2}-w_{n} d_{n}^{2}\right) H_{2 n}(\tau) \\
& +\frac{b c U}{2} w_{n-1} e_{n-1} d_{n-1} H_{2 n-2}(\tau)+E_{2 n} G_{2 n+2}(\tau) \\
& +\frac{b c U}{2} w_{n} d_{n} e_{n} H_{2 n+2}(\tau), n \geq 2
\end{aligned}
$$


and $A_{2 n}, E_{2 n}, w_{n}, e_{n}, d_{n}$ are constants.

In Figure 24 and Figure 25, we draw streamlines for the stream function $\psi_{a}$ in the plane $x_{2}=0$ with axes ratio $\frac{5}{3}$ and $\frac{10}{3}$.

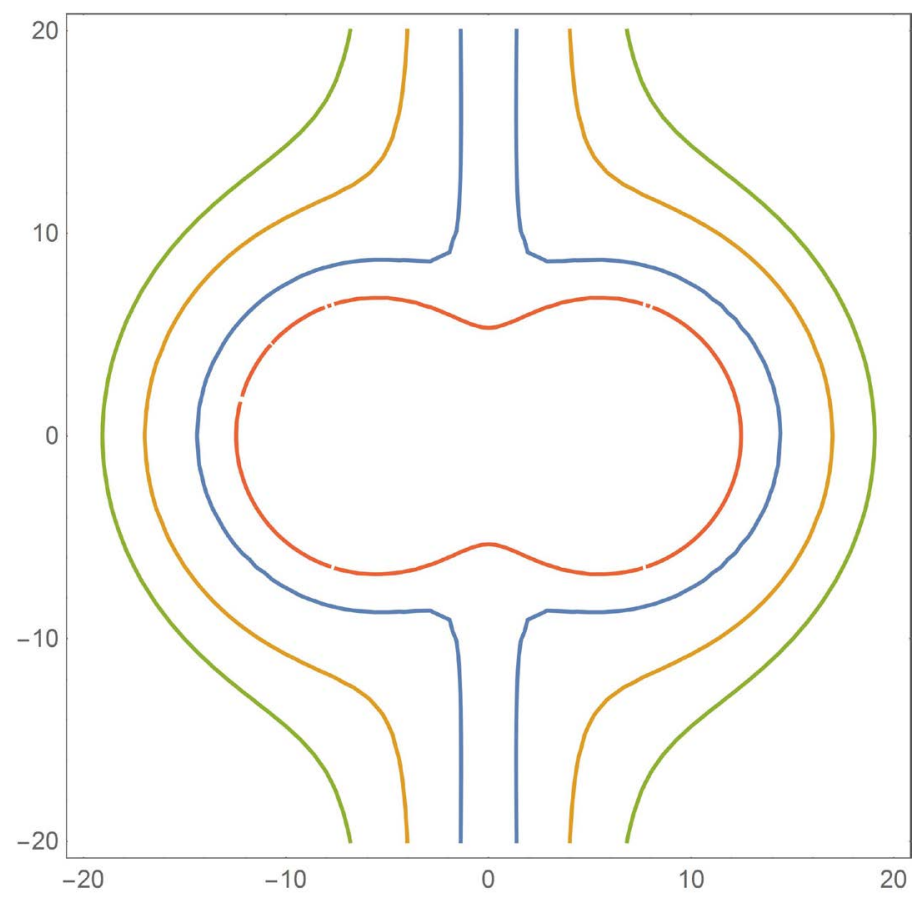

Figure 24. Streamlines for $\psi_{a}$ in the plane $x_{2}=0$ with axes ratio $\frac{5}{3}$.

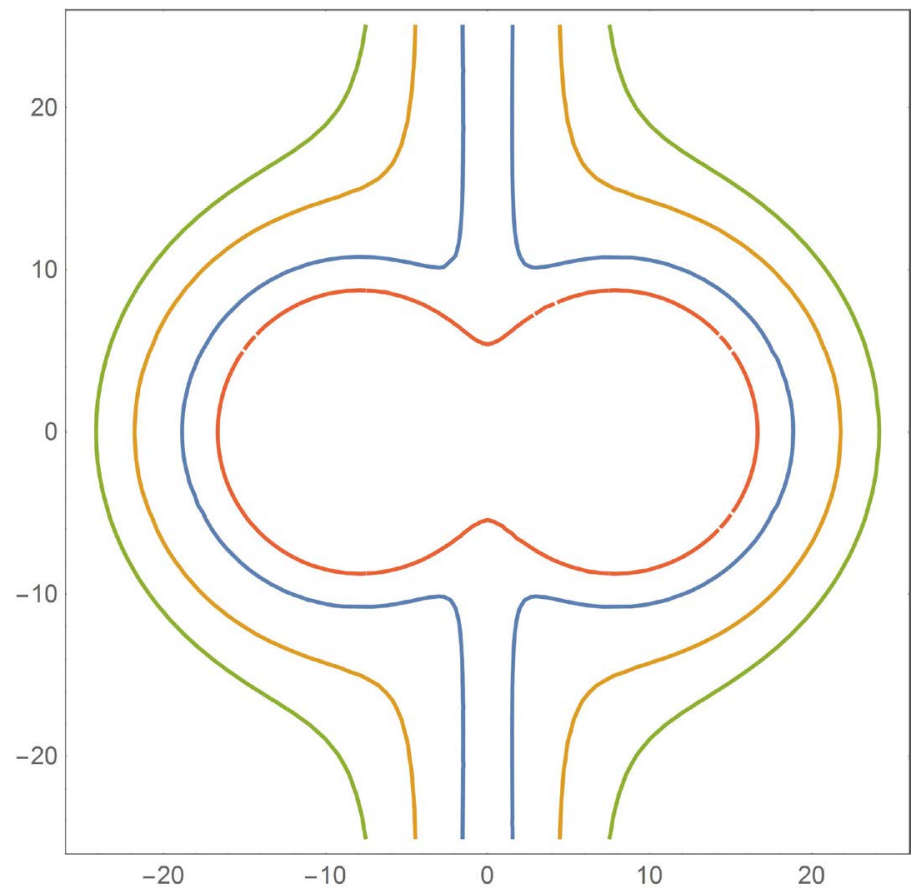

Figure 25. Streamlines for $\psi_{a}$ in the plane $x_{2}=0$ with axes ratio $\frac{10}{3}$. 
Using the stream function [31] analytical expressions for the drag force and the drag coefficient were also derived. Moreover, using the same methodology, the problem of the translation of a red blood cell through blood's plasma was solved [26], though which the derivation of the terminal settling velocity of the $\mathrm{RBC}$ was enabled.

\subsection{Blood's Plasma Flow Past a Swarm of Red Blood Cells}

Expanding the previous ideas and the particle-in-cell model [6], a mathematical model [32] that describes the flow of blood's plasma through a swarm of red blood cells (Figure 26) was developed. Particularly, the internal inverted prolate spheroid $\left(S_{a}^{\prime}\right)$ is assumed to be solid, while a fictitious external one $\left(S_{\beta}^{\prime}\right)$ circumscribes the fluid (Figure 27). The dimensions of the external spheroid are calculated such that the solid volume fraction in the cell equals to the solid volume fraction of the swarm [32].

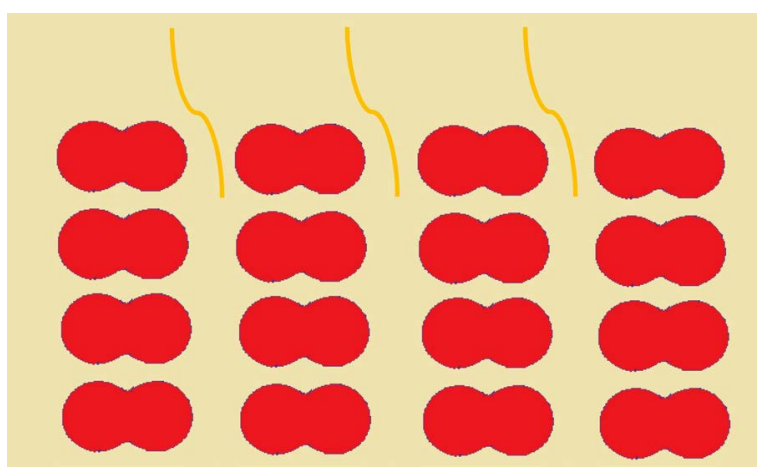

Figure 26. Swarm of RBCs in blood's plasma.

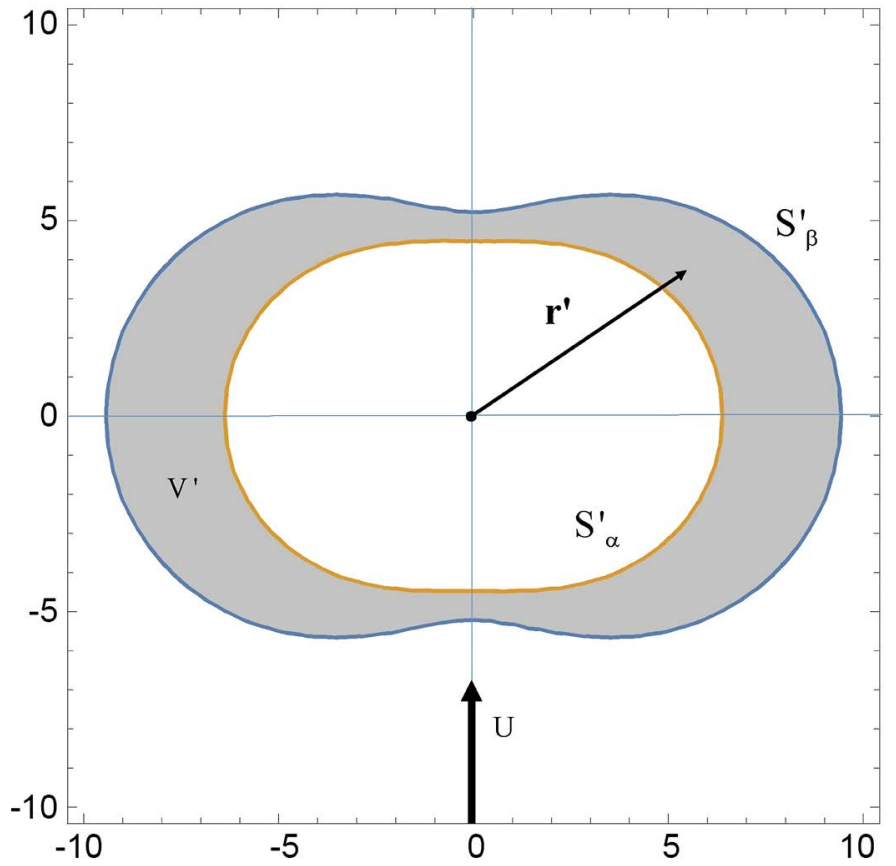

Figure 27. Statement of the problem. 
The problem at hand is mathematically formulated with the Equation (66) through (70)

$$
\begin{aligned}
& E^{\prime 4} \psi_{q}\left(r^{\prime}\right)=0, r^{\prime} \in V^{\prime}, \\
& \psi_{q}\left(r^{\prime}\right)=0, r^{\prime} \in \partial V_{a}^{\prime}, \\
& \frac{\partial \psi_{q}\left(r^{\prime}\right)}{\partial n}=0, r^{\prime} \in \partial V_{a}^{\prime}, \\
& \psi_{q}\left(r^{\prime}\right)=0, r^{\prime} \in \partial V_{\beta}^{\prime}, \\
& E^{\prime 2} \psi_{q}\left(r^{\prime}\right)=0, r^{\prime} \in \partial V_{\beta}^{\prime},
\end{aligned}
$$

where $E^{\prime 2}$ is the Stokes operator in $V^{\prime}, E^{\prime 4}=E^{\prime 2} O E^{\prime 2}$ is the Stokes bisteam operator and $\psi_{q}\left(r^{\prime}, \theta^{\prime}, \phi\right)$ stands for the stream function.

The analytic solution of the problem is given in [32] which is

$$
\psi_{q}(\tau, \zeta)=\frac{b^{3}}{r^{3}} \sum_{n=1}^{+\infty} g_{2 n}^{*}(\tau) G_{2 n}(\zeta)
$$

where $g_{n}^{*}$ are sums of Gegenbauer functions $G_{n}, H_{n}$ given analytically in [32].

In Figure 28 and Figure 29, we depict streamlines for $\psi_{q}\left(\tau^{\prime}, \zeta^{\prime}\right)$ using only the first term of the series solution, assuming the values $-0.3,-1,-4,-8,-12$ (from the outer to the inner inverted prolate spheroid). At this point, it is worth noticing that the second term of the series provides only small arithmetic corrections [32].

By employing the obtained expression for the stream function $\psi_{q}(\tau, \zeta)$, using (8), (9) expressions for the velocity components and the pressure field were also derived.

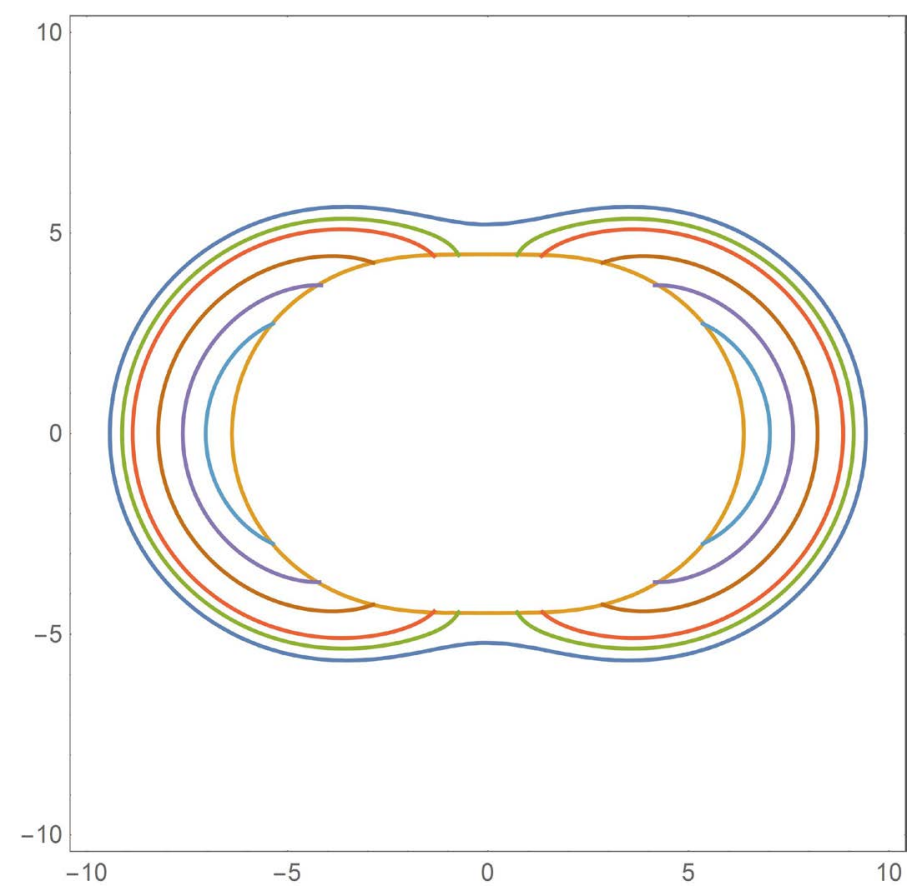

Figure 28. Streamlines for $\psi_{q}^{(2)}$ with $\tau_{a}=1.15$ in the plane $x_{2}=0$. 


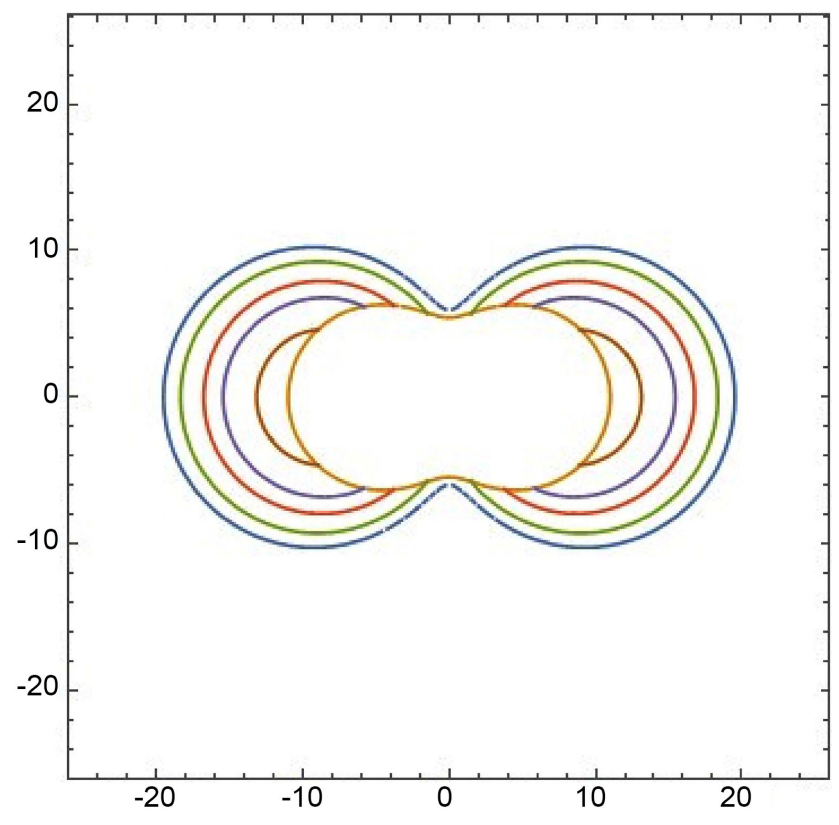

Figure 29. Streamlines for $\psi_{q}^{(2)}$ with $\tau_{\beta}=1.05$ in the plane $x_{2}=0$.

\subsection{Relative Motion of Blood's Plasma Flow Past Two Aggregated Low Density Lipoproteins}

The aggregation of low density lipoproteins (LDLs) is important in atherosclerosis, which is a decease that decreases the diameter of the arteries and increases blood pressure [28]. The two aggregated LDLs resemble an inverted oblate spheroid and due to the physical characteristics we model the flow as Stokes flow around an inverted oblate spheroid (Figure 30 ). The problem is defined through (72) to (75).

$$
\begin{gathered}
E^{\prime 4} \psi_{t}\left(\boldsymbol{r}^{\prime}\right)=0, \boldsymbol{r}^{\prime} \in V^{\prime}, \\
\psi_{t}\left(\boldsymbol{r}^{\prime}\right)+\frac{1}{2} \varpi^{\prime 2} U=0, \boldsymbol{r}^{\prime} \in \partial V^{\prime}, \\
\frac{\partial}{\partial n}\left[\psi_{t}\left(\boldsymbol{r}^{\prime}\right)+\frac{1}{2} \varpi^{\prime 2} U\right]=0, \boldsymbol{r}^{\prime} \in \partial V_{a}^{\prime}, \\
\frac{\psi_{t}\left(\boldsymbol{r}^{\prime}\right)}{r^{\prime 2}} \rightarrow 0, r^{\prime} \rightarrow+\infty,
\end{gathered}
$$

where $\psi_{t}$ is the stream function, (72) is the governing equation of the flow, (73) denotes that there is no relative tangential velocity component on the surface of the aggregated LDLs, (74) implies that the aggregated LDLs are impenetrable and (75) expresses the assumption that the blood plasma extends to infinity where it is at rest.

The solution [28] of the problem defined in (72)-(75) is

$$
\psi_{t}(\lambda, \tau)=\frac{b^{3}}{a^{3} \sqrt{\lambda^{2}-\tau^{2}+1}} \sum_{n=1}^{+\infty}\left[A_{2 n} G_{2 n}(i \lambda)+E_{2 n} G_{2 n+2}(i \lambda)\right] G_{2 n}(\tau),
$$

where $A_{2 n}, E_{2 n}$ are constants defined in [28]. 


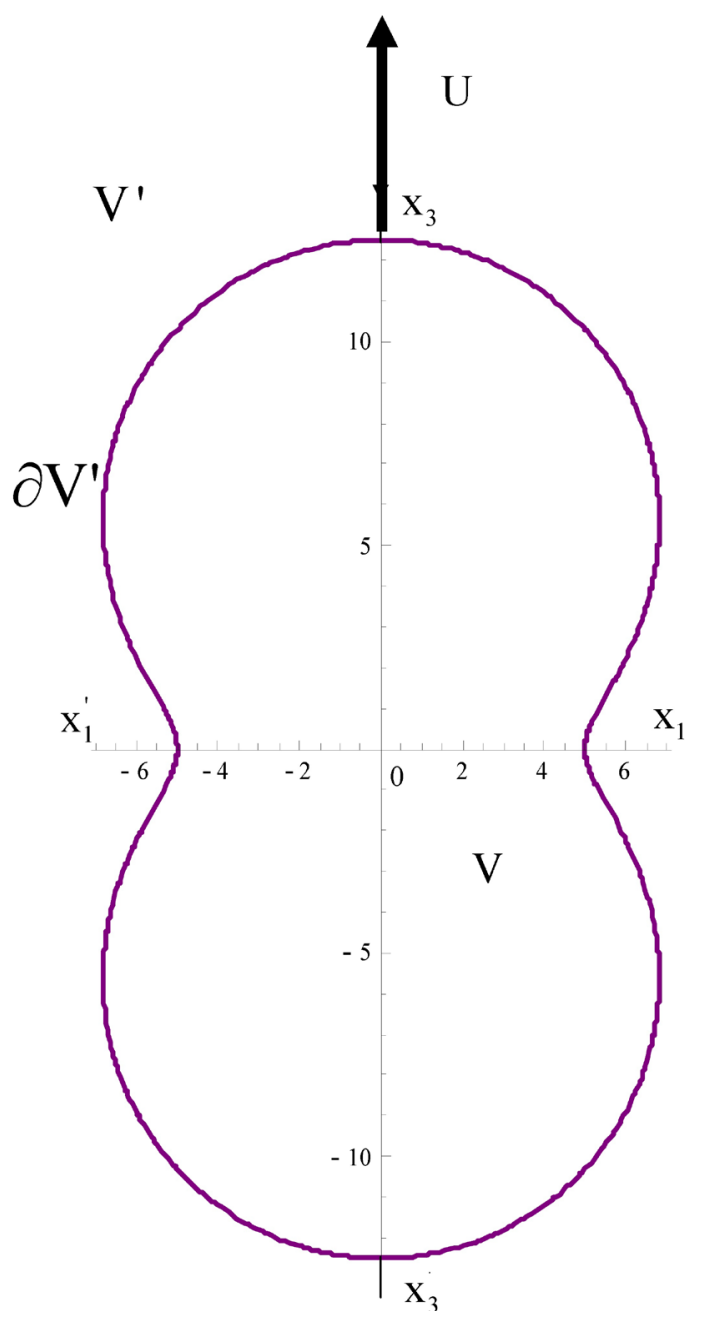

Figure 30. Statement of the problem.

\section{Conclusion}

The general solution for the Stokes axisymmetric flow equations $E^{2} \psi=0$ (irrotational) and $E^{4} \psi=0$ (rotational) are given in different separable forms of the corresponding eigenfunctions and generalized eigenfunctions, in terms of linear combinations of products of special functions. These are separable, $\mathrm{R}$-separable, semiseparable and R-semiseparable solutions. Each component in the series expansion of the analytical solution exhibits particular patterns, revealing physical and geometrical characteristics of the axisymmetric flow. In this manuscript, we collect, categorize, analyze and present in a systematic and comprehensive way relative results. The different kinds of the separation of variables that the Stokes operator can get in different axisymmetric systems are given in what follows. Emphasis is given in the qualitative results, while the reader is redirected to the original papers for the complete solution expansions. In the spherical [5], the parabolic [30] and the spheroidal coordinate system [6] $\left(q_{1}, q_{2}, \phi\right)$, the Stokes equation separates variables, and the stream function $\psi\left(q_{1}, q_{2}\right)$ can be written as a sum of products of two functions of one single va- 
riable each one, denoting the radial and the angular dependence $q_{1}, q_{2}$, respectively. This kind of separability is considered as a $1 \mathrm{D}$ by $1 \mathrm{D}$ decomposition of the kernel space of the Stokes operator $E^{2}$. Furthermore, Stokes equation in the bispherical, toroidal [22], inverted spheroidal [24] [25], tangent sphere and cardioid geometries [30] R-separates variables, and the stream function is given as product of a simple series expansion with a function $\frac{1}{R\left(q_{1}, q_{2}\right)}$, where $R\left(q_{1}, q_{2}\right)$ can not be written in a separable form. Moreover, all the necessary conditions for the simple separability or the R-separability of the Stokes operator as formulated, stated and proved in [3], are also presented, providing accurate criteria and ready to use results for those who seek for analytical solutions for the rotational and irrotational Stokes flows, in any axisymmetric coordinate system. Taking into account that the eigenfunctions of the kernel of the $E^{2}$ operator, form a complete set of solutions for the irrotational flow in the corresponding geometry, their derivation is a necessary step for obtaining a solution of the irrotational Stokes flow, $E^{4} \psi=0$, where $E^{4}=E^{2} O E^{2}$. It was shown that in the spherical coordinate system, due to the symmetry to any direction, Stokes bi-stream operator $E^{4}$ separates variables [5], while in spheroidal coordinates, due to the axis symmetry [6], it semiseparates variables. In the inverted spheroidal coordinate systems [24] [25] $E^{2} \psi=0$ R-separates variables, which reflects the geometrical inversion (with respect to a sphere) of the coordinate system to the analytical solution. The generalized eigenfunctions of Stokes operator $E^{2}$ in the spheroidal geometry are obtained in terms of a 3D by $3 \mathrm{D}$ combinations of Gegenbauer functions for each variable, of two kinds and of $n, n-2, n+2$ degree, justifying the notion of semiseparation, while accordingly, their inverted ones are multiplied by the Euclidean distance on the minus third, $r^{-3}$. This decomposition is denoted as $\mathrm{R}$-semiseparation. The stream function obtained this way, is sufficient general to be applied to interior and exterior boundary value problems and has been employed for solving boundary value problems arising in various scientific fields. We demonstrate, indicatively, applications in Biology, concerning the modeling and the study of the relative motion of blood plasma flow past a red blood cell or a swarm of red blood cells and also the problem of blood plasma flow past two aggregated low density lipoproteins. The obtained analytical expansions for the stream function can be used for deriving other physical quantities of interest such as the velocity and the pressure filed. They may also be used as basis for numerical implementation.

\section{Conflicts of Interest}

The authors declare no conflicts of interest regarding the publication of this paper.

\section{References}

[1] Moon, P. and Spencer, E.D. (1961) Field Theory Handbook. Springer-Verlag, Berlin. https://doi.org/10.1007/978-3-642-53060-9 
[2] Morse, M.P. and Feshbach, H. (1953) Methods of Theoretical Physics. McGraw-Hill, New York.

[3] Hadjinicolaou, M. and Protopapas, E. Necessary and Sufficient Conditions for the Separability and the R-Separability of the Stokes Equation. (Submitted for Publication)

[4] Stokes, G.G. (1851) On the Effect of the Internal Friction of Fluids on the Motion of Pendulums. Transactions of the Cambridge Philosophical Society, 8, 8-106.

[5] Happel, J. and Brenner, H. (1991) Low Reynolds Number Hydrodynamics. Kluwer Academic Publishers, Dordrecht, The Netherlands.

[6] Dassios, G., Hadjinicolaou, M. and Payatakes, A.C. (1994) Generalized Eigenfunctions and Complete Semiseparable Solutions for Stokes Flow in Spheroidal Coordinates. Quarterly of Applied Mathematics, LII, 157-191.

https://doi.org/10.1090/qam/1262325

[7] Dassios, G., Hadjinicolaou, M., Coutelieris, A.F. and Payatakes, A.C. (1995) Stokes Flow in Spheroidal Particle in Cell Models with Happel and Kuwabara Boundary Conditions. International Journal of Engineering Science, 33, 1465-1490.

https://doi.org/10.1016/0020-7225(95)00010-U

[8] Dassios, G., Hadjinicolaou, M. and Protopapas, E. (2012) Blood Plasma Flow Past a Red Blood Cell: Mathematical Modelling and Analytical Treatment. Mathematical Methods in the Applied Sciences, 35, 1547-1563. https://doi.org/10.1002/mma.2540

[9] Sampson, A.R. (1891) On the Stoke's Current Function. Philosophical Transactions of the Royal Society A, 182, 449. https://doi.org/10.1098/rsta.1891.0012

[10] Kim, S. (1987) Stokes Flow Past Three Spheres: An Analytic Solution. Physics of Fluids, 30, 2309. https://doi.org/10.1063/1.866120

[11] Sankar, P.N. (2009) Exact Solutions for Stokes Flow in and around a Sphere and between Concentric Spheres. Journal of Fluid Mechanics, 631, 363-373. https://doi.org/10.1017/S0022112009007265

[12] Oberbeck, A. (1876) Ueber Stationäre Flüssigkeitsbewegungen mit Berücksichtigung der inneren Reibung. Journal für die reine und angewandte Mathematik, 81, 62-80. https://doi.org/10.1515/crll.1876.81.62

[13] Payne, L.E. and Pell, W.H. (1960) The Stokes Flow Problem for a Class of Axially Symmetric Bodies. Journal of Fluid Mechanics, 4, 529-549. https://doi.org/10.1017/S002211206000027X

[14] Coutelieris, F.A., Burganos, V.N. and Payatakes, A.C. (1995) Convective Diffusion and Adsorption in a Swarm of Spheroidal Particles. AIChE Journal, 41, 1122-1134. https://doi.org/10.1002/aic.690410508

[15] Coutelieris, F.A., Burganos, V.N. and Payatakes, A.C. (2004) Model of Adsorption-Reaction-Desorption in a Swarm of Spheroidal Particles. AIChE Journal, 50, 779-785. https://doi.org/10.1002/aic.10072

[16] Keh, H.J. and Chang, Y.C. (2009) Thermophoresis of an Aerosol Spheroid along Its Axis of Revolution. Physics of Fluids, 21, Article ID: 062001. https://doi.org/10.1063/1.3156002

[17] Keh, H.J. and Chang, Y.C. (2008) Slow Motion of a Slip Spheroid along Its Axis of Revolution. International Journal of Multiphase Flow, 34, 713-722. https://doi.org/10.1016/j.ijmultiphaseflow.2008.02.002 
[18] Zlatanovski, T. (1999) Axisymmetric Creeping Flow Past a Porus Prolate Spheroidal Particle Using the Brinkman Model. The Quarterly Journal of Mechanics and Applied Mathematics, 52, 111-126. https://doi.org/10.1093/qjmam/52.1.111

[19] Deo, S. and Datta, S. (2003) Stokes Flow Past a Fluid Prolate Spheroid. Indian Journal of Pure and Applied Mathematics, 34, 755-764.

[20] Deo, S. and Gupta, B.R. (2009) Stokes Flow Past a Swarm of Porous Approximately Spheroidal Particles with Kuwabara Boundary Condition. Acta Mechanica, 203, 241-254. https://doi.org/10.1007/s00707-008-0048-0

[21] Dassios, G. and Vafeas, P. (2001) Connection Formulae for Differential Representations in Stokes Flow. Journal of Computational and Applied Mathematics, 133, 283-294. https://doi.org/10.1016/S0377-0427(00)00651-8

[22] Deo, S. and Tiwari, A. (2008) On the Solution of a Partial Differential Equation Representing Irrotational Flow in Bispherical Polar Coordinates. Applied Mathematics and Computation, 205, 475-477. https://doi.org/10.1016/j.amc.2008.08.023

[23] Hadjinicolaou, M. and Protopapas, E. (2013) On the R-Semiseparation of the Stokes Bi-Stream Operator in the Inverted Prolate Spheroidal Coordinates. Mathematical Methods in the Applied Sciences, 37, 207-211. https://doi.org/10.1002/mma.2841

[24] Hadjinicolaou, M. and Protopapas, E. (2015) Spectral Decomposition of the Stokes Flow Operators in the Inverted Prolate Spheroidal Coordinates. The IMA Journal of Applied Mathematics, 80, 1475-1491. https://doi.org/10.1093/imamat/hxv003

[25] Hadjinicolaou, M. and Protopapas, E. (2016) Eigenfunction Expansions for the Stokes Flow Operators in the Inverted Oblate Coordinate System. Mathematical Problems in Engineering, 2016, Article ID: 9049131. https://doi.org/10.1155/2016/9049131

[26] Hadjinicolaou, M., Kamvyssas, G. and Protopapas, E. (2013) Stokes Flow Applied to the Sedimentation of a Red Blood Cell. Quarterly of Applied Mathematics.

[27] Hadjinicolaou, M. (2015) A Mathematical Model for the Blood Plasma Flow around Two Aggregated Low Density Lipoproteins. Advanced Experimental Medicine and Biology, 820, 173-184. https://doi.org/10.1007/978-3-319-09012-2_11

[28] Hadjinicolaou, M. and Protopapas, E. (2015) Translation of Two Aggregated Low Density Lipoproteins within Blood Plasma. A Mathematical Model. Advanced EXperimental Medicine and Biology, 820, 185-192. https://doi.org/10.1007/978-3-319-09012-2_12

[29] Lebedev, M.N. (1972) Special Functions and Their Applications. Dover Publications, New York.

[30] Protopapas, E. On the Solution of Irrotational Stokes Flow in Rotational System of Coordinates. (Submitted for Publication)

[31] Hadjinicolaou, M. and Protopapas, E. (2012) Studying the Blood Plasma Flow Past a Red Blood Cell, with the Mathematical Method of Kelvin's Transformation. 12th International Conference on Bioinformatics \& Bioengineering, Larnaca, Cyprus, 11-13 November 2012, 662-665. https://doi.org/10.1109/BIBE.2012.6399745

[32] Hadjinicolaou, M. and Protopapas, E. (2020) A Microscale Mathematical Blood Flow Model for Understanding Cardiovascular Diseases. Springer, New York. 


\section{Nomenclature}

\begin{tabular}{|c|c|c|c|}
\hline$\psi$ & stream function & $r$ & position vector \\
\hline$E^{2}$ & irrotational Stokes operator & $E^{4}$ & rotational Stokes operator \\
\hline$v(r)$ & velocity field & $P(\boldsymbol{r})$ & pressure field \\
\hline$\mu$ & shear viscosity & $u_{q_{1}}, u_{q_{2}}$ & velocity components \\
\hline$\left(q_{1}, q_{2}, \varphi\right)$ & axisymmetric coordinate system & $F_{z}$ & drag force \\
\hline$\rho\left(q_{1}, q_{2}\right)$ & circular cylindrical coordinate & $z\left(q_{1}, q_{2}\right)$ & circular cylindrical coordinate \\
\hline$h_{1}, h_{2}$ & metric coefficients & $\varpi$ & radial cylindrical coordinate \\
\hline$\left(x_{1}, x_{2}, x_{2}\right)$ & cartesian coordinates & $C_{d}$ & drag coefficient \\
\hline$U$ & particle speed & $A$ & cross sectional area \\
\hline$\rho$ & the fluid density & $U_{\infty}$ & settling terminal velocity \\
\hline$\rho^{\prime}$ & the mean particle density & $g$ & local acceleration of gravity vector \\
\hline$V$ & particle's volume & $G_{n}, H_{n}$ & Gegenbauer functions of $n$-th degree \\
\hline$(r, \zeta, \phi)$ & spherical coordinate system & $(\mu, v, \varphi)$ & cardioid coordinate system \\
\hline$(\tau, \zeta, \phi)$ & prolate coordinate system & $c$ & semifocal distance in prolate geometry \\
\hline$(\lambda, \zeta, \varphi)$ & oblate coordinate system & $a$ & semifocal distance in oblate geometry \\
\hline$(\eta, \theta, \varphi)$ & bispherical coordinate system & $(\eta, \theta, \varphi)$ & toroidal coordinate system \\
\hline$(\mu, v, \varphi)$ & parabolic coordinate system & $(\mu, v, \varphi)$ & tangent sphere coordinate system \\
\hline $\begin{array}{c}Y_{1}, K_{1} \\
\psi_{a}\left(\boldsymbol{r}^{\prime}\right)\end{array}$ & \multicolumn{3}{|c|}{ stream function for blood plasma flow past a RBC } \\
\hline$\psi_{q}\left(\boldsymbol{r}^{\prime}\right)$ & \multicolumn{3}{|c|}{ stream function for blood plasma flow past a swarm of RBCs } \\
\hline$\psi_{t}\left(\boldsymbol{r}^{\prime}\right)$ & stream function for blood plasma flo & ow past tw & To aggregated LDLs \\
\hline
\end{tabular}

